\title{
Baylis-Hillman Mechanism: A New Interpretation in Aprotic Solvents
}

Price, K.E.; Broadwater, S.J.; Jung, H.M.; McQuade, D.T.

\begin{tabular}{lc}
\hline Title & Page \\
\hline Experimental Methods & 1 \\
Order Plots & 3 \\
KIE at $\alpha$ position of methyl acrylate & 31 \\
Aldehyde Proton KIE & 38 \\
High Conversion NMR Spectrum & 44
\end{tabular}

General Procedures. Materials were obtained from Aldrich and Acros and used without further purification, unless otherwise noted. Solvents and aldehydes were purified by standard procedures. 4-Pyridine carboxaldehyde was used immediately after purification. ${ }^{1} \mathrm{H}$ - and ${ }^{13} \mathrm{C}$-NMR spectra were obtained on Varian Mercury $300 \mathrm{MHz}$, Varian Inova 400 $\mathrm{MHz}$, and Varian Inova $500 \mathrm{MHz}$ spectrometers, with solvent acting as the internal reference. Gas chromatographic (GC) analyses were carried out on a Varian Model 3800 using a CP-Sil regular phase column (30.0 m x $0.25 \mathrm{~mm}$ i.d.). Peak areas were measured using the Varian Star 6.2 software package, and response factors of authentic materials versus methyl benzoate (internal standard) were calculated for determining reaction conversion.

Preparation of $\boldsymbol{\alpha}$-deuterio methyl acrylate: The preparation was modified from that of Baldwin and Cianciosi. ${ }^{1}$ Diazabicyclo[2.2.2] octane $(25 \mathrm{~g}, 0.22 \mathrm{~mol})$ was dissolved in methanol-D $(108 \mathrm{~mL}, 2.65 \mathrm{~mol})$ with stirring. Methyl acrylate $(20 \mathrm{~mL}, 0.22 \mathrm{~mol})$ was added and the solution was stirred at room temperature for approximately 12 hours. The solution was then diluted in $o$-dichlorobenzene $(\mathrm{ODCB})$ and washed with water $(\mathrm{x} 4)$ and brine (x1). The organic phase was dried over $\mathrm{Na}_{2} \mathrm{SO}_{4}$ and fractionally distilled to remove the d-methyl acrylate from the ODCB. The d-methyl acrylate was isolated as a colorless liquid $(7 \mathrm{~g}, 36 \%)$ and contained less than $2 \%$ ODCB as determined by ${ }^{1} \mathrm{H}-\mathrm{NMR}$ spectroscopy. This volume difference was accounted for when running d-methyl acrylate kinetics. The d-methyl acrylate was approximately $87 \%$ deuterated and was stored cold over $\mathrm{Na}_{2} \mathrm{SO}_{4} .{ }^{1} \mathrm{H}-\mathrm{NMR}\left(300 \mathrm{MHz}, \mathrm{CDCl}_{3}\right): \delta 6.40(\mathrm{~m}, 1 \mathrm{H}), 6.12(\mathrm{~m}, 0.15 \mathrm{H}), 5.83(\mathrm{~m}$, $1 \mathrm{H}), 3.75$ (s, 3H).

Preparation of C,C-dideuterio-C-(4-nitrophenyl)-methanol. 4-Nitrobenzoyl chloride $(8.0 \mathrm{~g}, 43.1 \mathrm{mmol})$ was dissolved in dry THF $(200 \mathrm{~mL})$ and added dropwise to a suspension of lithium aluminum deuteride $(2.2 \mathrm{~g}, 51.8 \mathrm{mmol})$ in THF $(100 \mathrm{~mL})$ at $-78^{\circ} \mathrm{C}$ over 2 hours. The reaction mixture was allowed to stir for an additional hour at $-78^{\circ} \mathrm{C}$ and then allowed to warm to RT for 30 minutes. The reaction mixture was quenched with $1 \mathrm{~N} \mathrm{HCl}$, diluted with EtOAc and filtered. The filtrate was washed with brine $(3 \times 50 \mathrm{~mL})$ and water $(3 \mathrm{x}$ $50 \mathrm{~mL})$, dried over $\mathrm{Na}_{2} \mathrm{SO}_{4}$, and concentrated to produce a yellow solid $(70 \%, 4.7 \mathrm{~g}) .{ }^{1} \mathrm{H}-$ NMR (400 MHz, acetone-d $\left.{ }_{6}\right): \delta 8.20(\mathrm{~d}, J=8.8 \mathrm{~Hz}, 2 \mathrm{H}), 7.58(\mathrm{~d}, J=8.8 \mathrm{~Hz}, 2 \mathrm{H}), 4.62$ (s, $1 \mathrm{H})$.

Preparation of $\boldsymbol{\alpha}$-deuterio-4-nitrobenzaldehyde. The material was prepared following the general procedure of Moore and Finney. ${ }^{2}$ C,C-dideuterio-C-(4-nitrophenyl)-methanol 
(4.3g, 27.7mmol), o-iodoxybenzoic acid (IBX) (23.3g, 83.2mmol), and EtOAc (200mL) were combined in a $500 \mathrm{~mL}$ round bottom flask equipped with a condenser. ${ }^{*}$ Caution: IBX can explode on impact or heating. ${ }^{3}$ The reaction mixture was stirred vigorously at $80^{\circ} \mathrm{C}$ overnight, open to atmosphere. The reaction mixture was cooled, filtered and concentrated to produce a yellow solid. The solid was purified by flash chromatography on silica (pre-loaded with $\mathrm{CH}_{2} \mathrm{Cl}_{2}$, eluted with 4:1 hexanes:EtOAc) to produce a yellow crystalline solid $(93 \%, 3.9 \mathrm{~g}) .{ }^{1} \mathrm{H}-\mathrm{NMR}\left(400 \mathrm{MHz}, \mathrm{CDCl}_{3}\right): \delta 8.38(\mathrm{~d}, J=8.8 \mathrm{~Hz}, 2 \mathrm{H})$, $8.08(\mathrm{~d}, J=8.8 \mathrm{~Hz}, 2 \mathrm{H}) .{ }^{2} \mathrm{H}-\mathrm{NMR}\left(500 \mathrm{MHz}, \mathrm{CHCl}_{3}\right.$ spiked with $\left.\mathrm{CDCl}_{3}\right): \delta 10.02$ (s, 1D).

Preparation of 2-[hydroxyl-(4-nitro-phenyl)-methyl]-acrylic acid methyl ester: This molecule was synthesized using the general kinetic procedure with dimethyl sulfoxide as the solvent. The spectroscopic characterization is consistent with published characterization. ${ }^{4}{ }^{1} \mathrm{H}-\mathrm{NMR}\left(500 \mathrm{MHz}, \mathrm{CDCl}_{3}\right): \delta 8.19(\mathrm{~d}, J=8.8 \mathrm{~Hz}, 2 \mathrm{H}), 7.56(\mathrm{~d}, J=$ $9.3 \mathrm{~Hz}, 2 \mathrm{H}), 6.38(\mathrm{~s}, 1 \mathrm{H}), 5.86(\mathrm{~s}, 1 \mathrm{H}), 5.62(\mathrm{~s}, 1 \mathrm{H}), 3.73(\mathrm{~s}, 3 \mathrm{H}) .{ }^{13} \mathrm{C}-\mathrm{NMR}(500 \mathrm{MHz}$, CDCl3): $\delta 166.36,148.52,147.41,140.85,127.31,127.29,123.61,72.74,52.22$.

\section{General Kinetic Procedure:}

All reactions were carried out with methyl acrylate, aldehyde, DABCO, and methyl benzoate at concentrations of $0.84 \mathrm{M}, 0.83 \mathrm{M}, 0.27 \mathrm{M}$, and $0.066 \mathrm{M}$, respectively. Reactions were started with the addition of methyl acrylate to a solution of aldehyde, $\mathrm{DABCO}$, and methyl benzoate in a given solvent. The reactions were monitored by diluting $5-7 \mu \mathrm{L}$ of the reaction mixture into $1.5 \mathrm{~mL} \mathrm{CH}_{2} \mathrm{Cl}_{2}$ at appropriate time intervals for GC analysis. For the acrylate, DABCO, and aldehyde order plots, the amount of DMSO and the reaction volume were held constant to eliminate solvent effects. THF was used as the non-reactive substitute for absent reagents. All reactions were monitored prior to $10 \%$ conversion. No byproducts were detected at high conversions. Relative rates were determined in Sigma Plot 8.0. Aberrant rates were eliminated using the Student's t-test at the $95 \%$ confidence interval.

1. Baldwin, J.E.; Cianciosi, S.J. J. Am. Chem. Soc.1992, 114 (24), 9401-9408.

2. Moore, J.D.; Finney, N.S. Org. Lett. 2002, 4 (17), 3001-3003.

3. Plumb, J.B.; Harper, D.J. Chem. Eng. News 1990, 68 (29), 3.

4. Shi, M.; Li, C.Q.; Jiang, J. K. Tetrahedron 2003, 59 (8), 1181-1189. 


\section{Baylis-Hillman Order and KIE Data:}

Notes: When examining Pdt/Std ratios, values over 1.05 were cut arbitrarily due to high conversion. Data that was removed with the t-test is not present in this data set. "Run 2" after the name of a reaction indicates that it was run on the GC multiple times. The points on the order plot that are representative of our standard kinetics conditions can be found with the kinetic isotope effect experiment data.

\section{Order Plots in DMSO}

A. Order in DABCO: The number of mols of THF+ DABCO were kept constant for each point so that the amount of DMSO would remain constant between the low and high concentration points.

Data Summary:

\begin{tabular}{rrr}
\hline [DABCO] & \multicolumn{1}{l}{ Rate } & \multicolumn{1}{c}{ Std Dev } \\
\hline 0.0838263 & 0.001232 & 0.000133 \\
0.1685637 & 0.00222 & 0.000226 \\
0.25239 & 0.0045 & 0.00034 \\
0.3371274 & 0.0058 & 0.000406
\end{tabular}

Order Plot:

Order in DABCO

$y=a x^{\wedge} b \quad a=0.0159 b=0.9948 r 2=0.976$

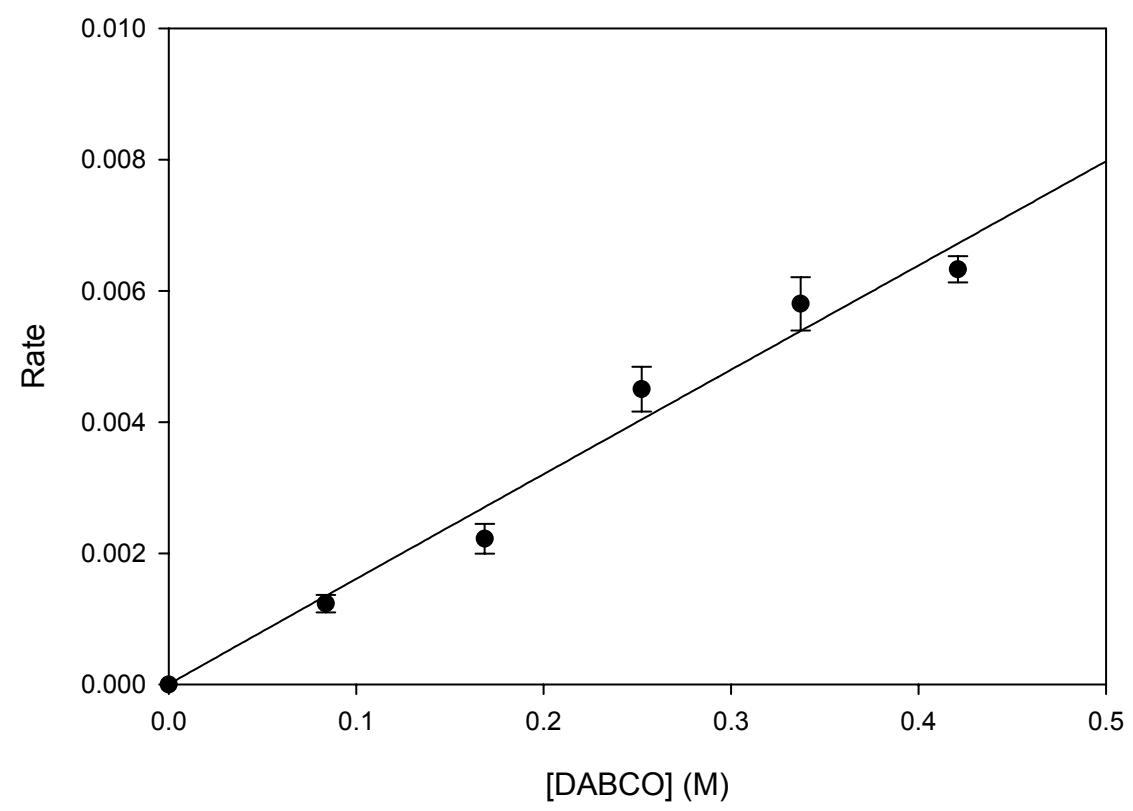




\section{1 eq DABCO}

Average Rate (no 6 on Run 3) $=0.001232 \mathrm{std} \mathrm{dev}=0.000133$
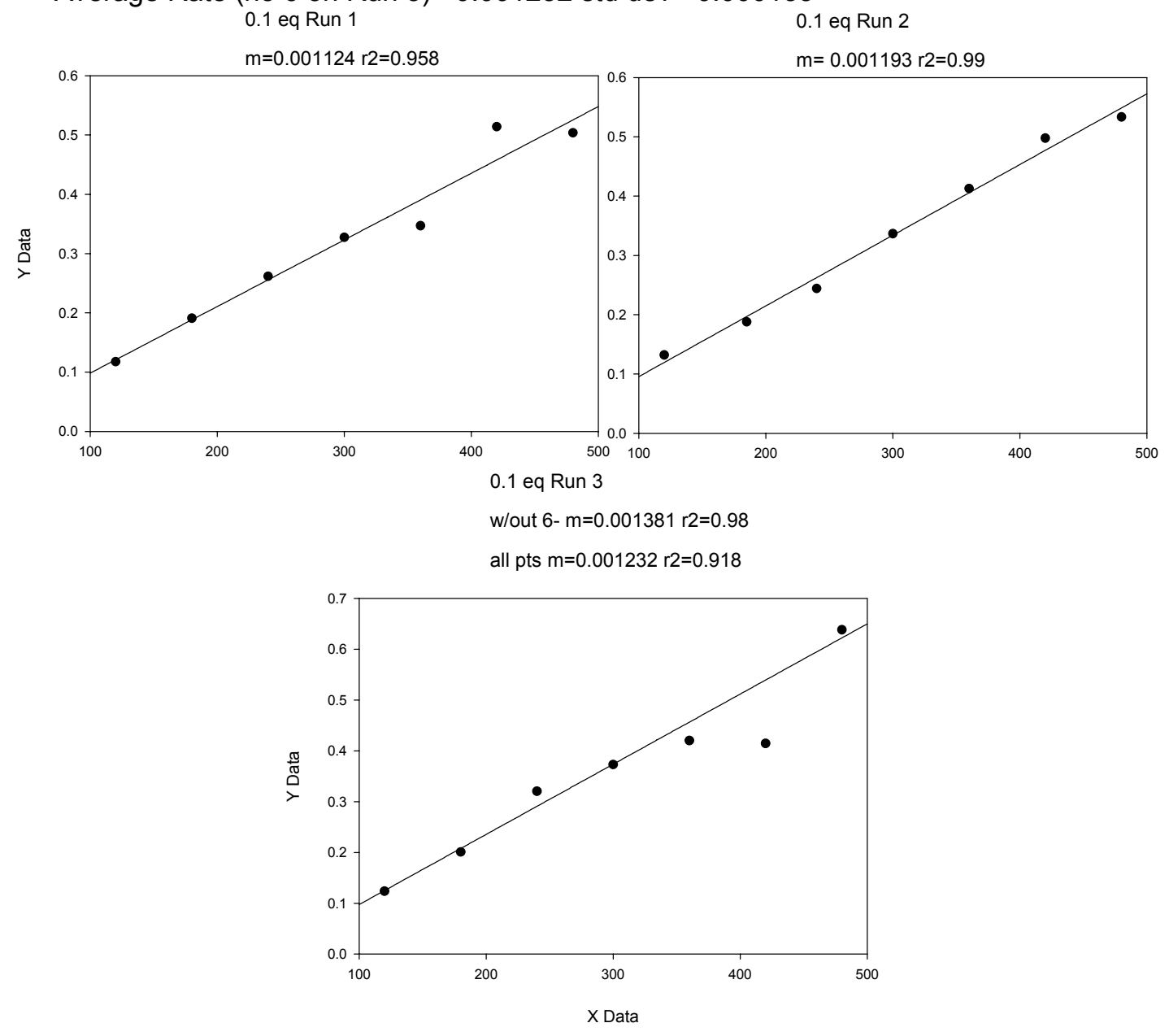


\section{2 eq DABCO Average Rate $=0.00222$ Std dev $=0.00023(10 \%)$}

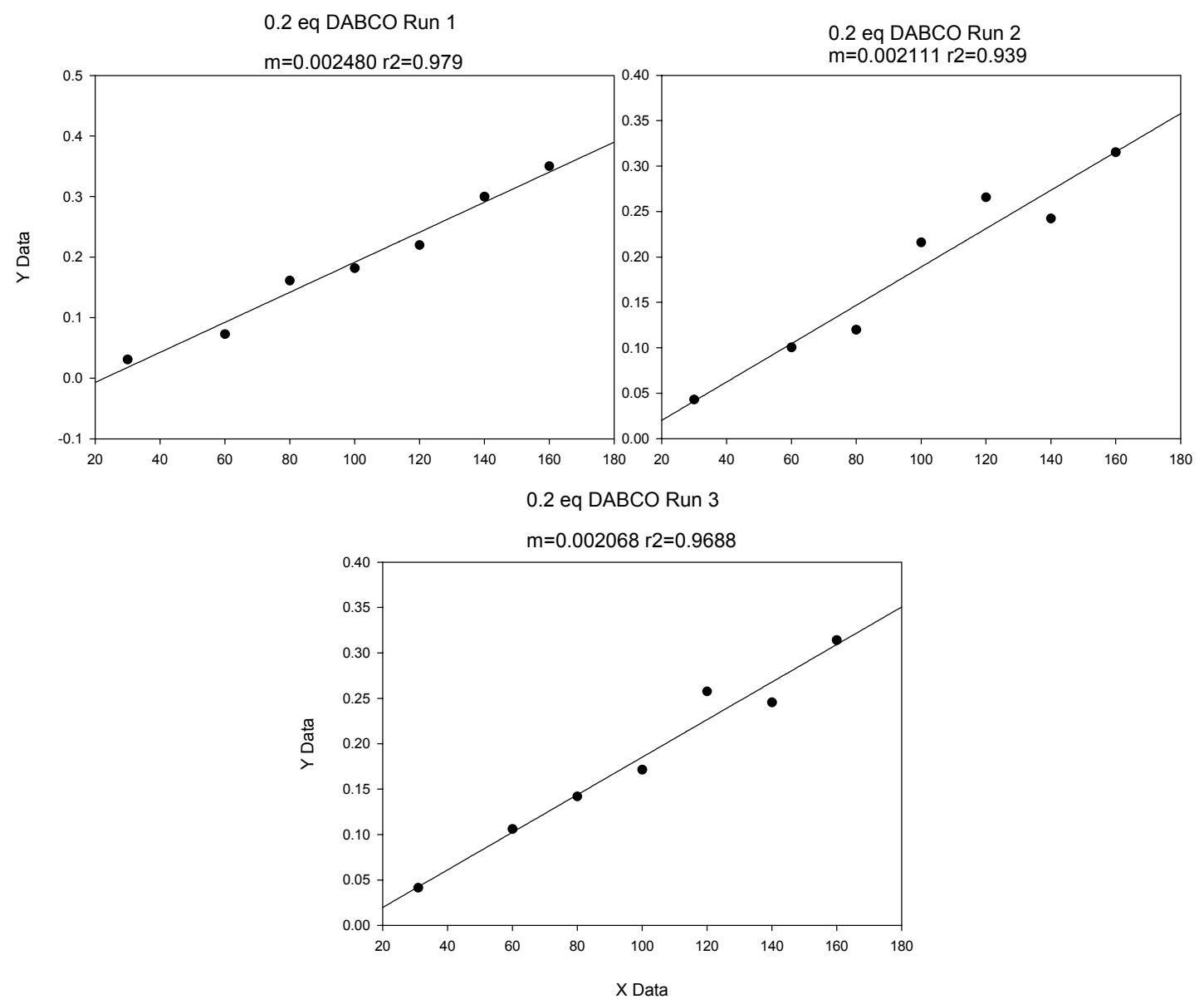




\section{3 eq DABCO}

Avg Rate 0.0045 std dev=0.00034 (7.6\%)
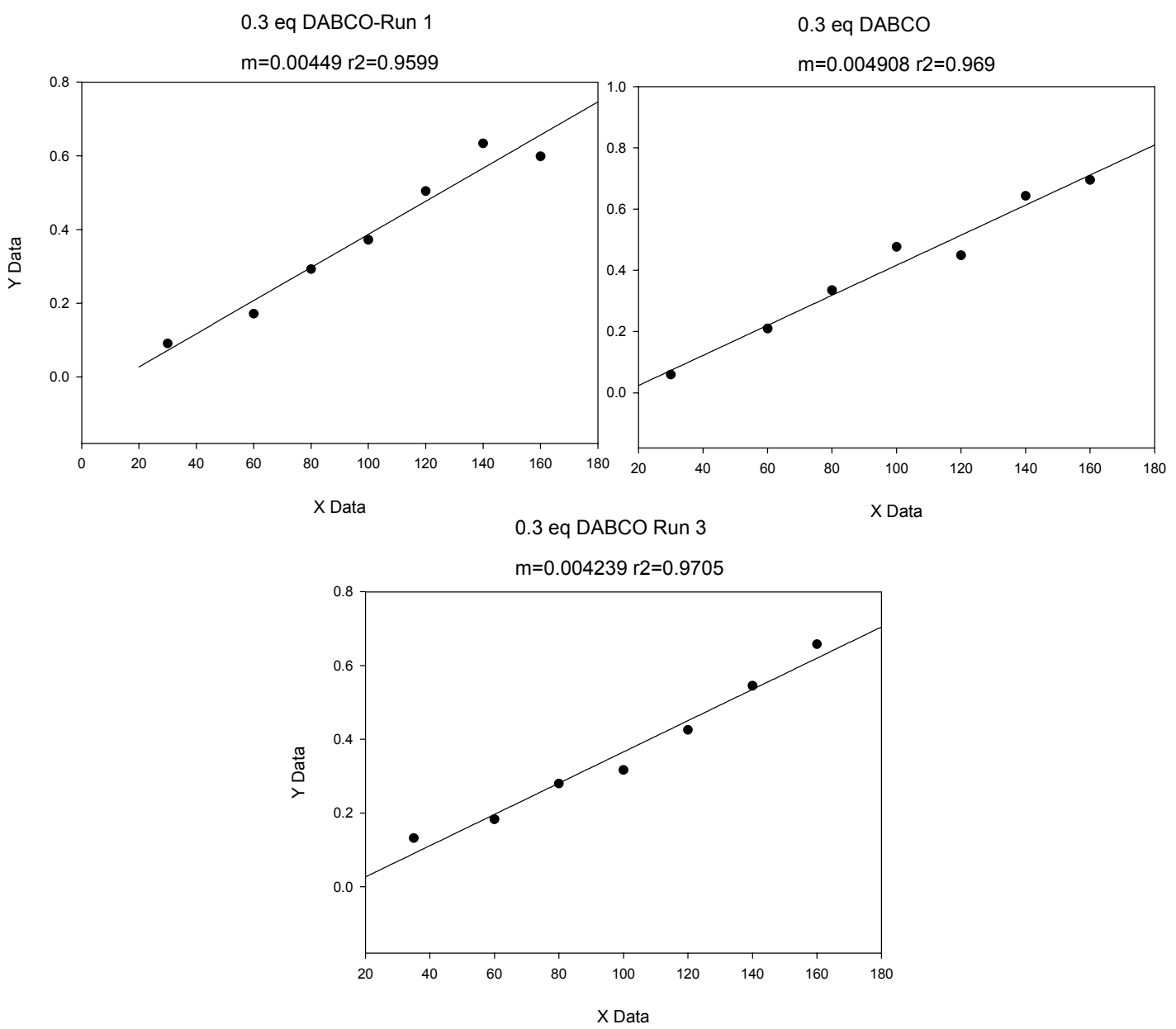
0.4 eq DABCO with THF as the filler avg rate $=0.0058$

std dev $=0.000406(7 \%)$
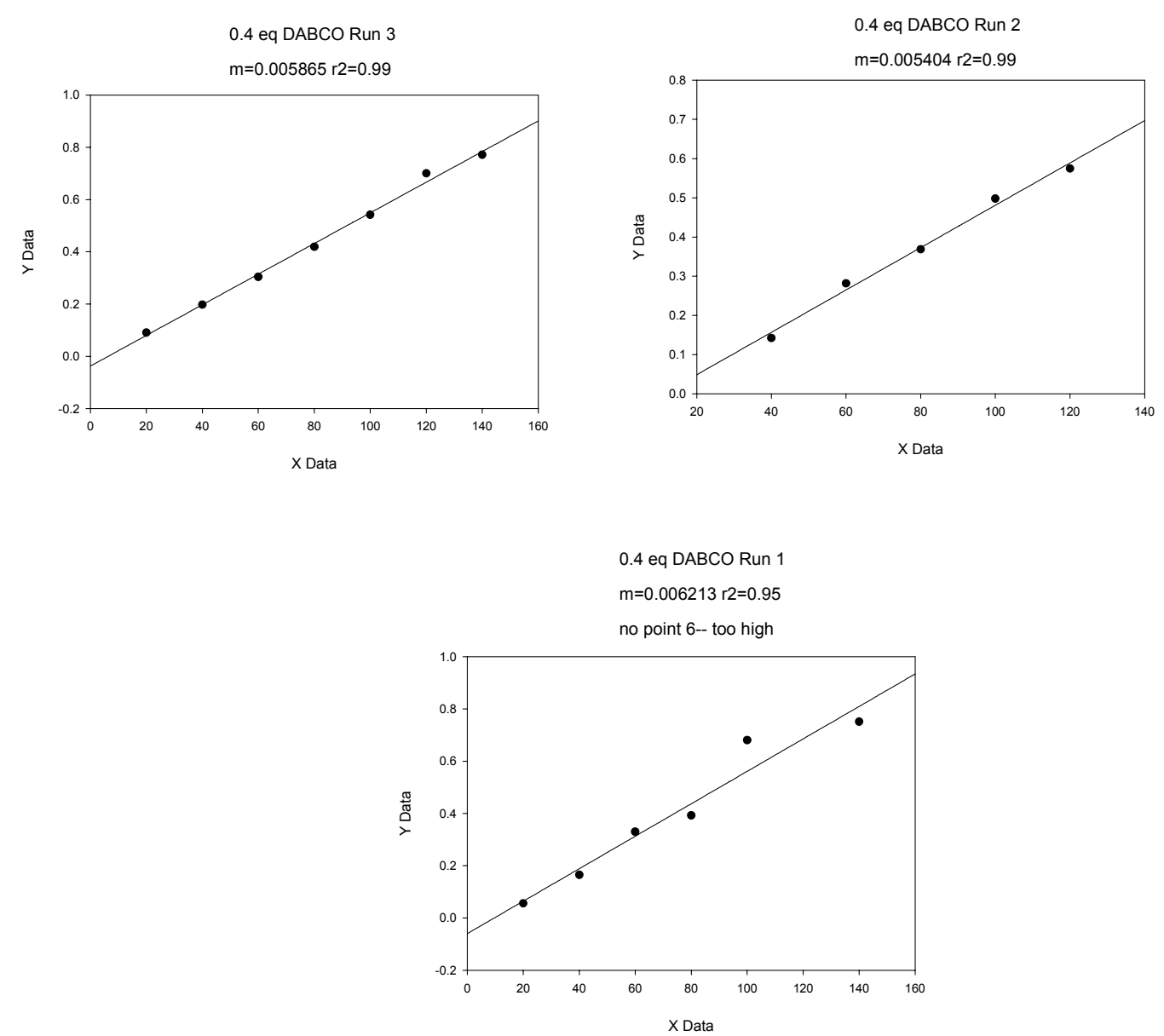
0.5 eq DABCO Average Rate $=0.006326$ Std dev $=0.0002(3 \%)$

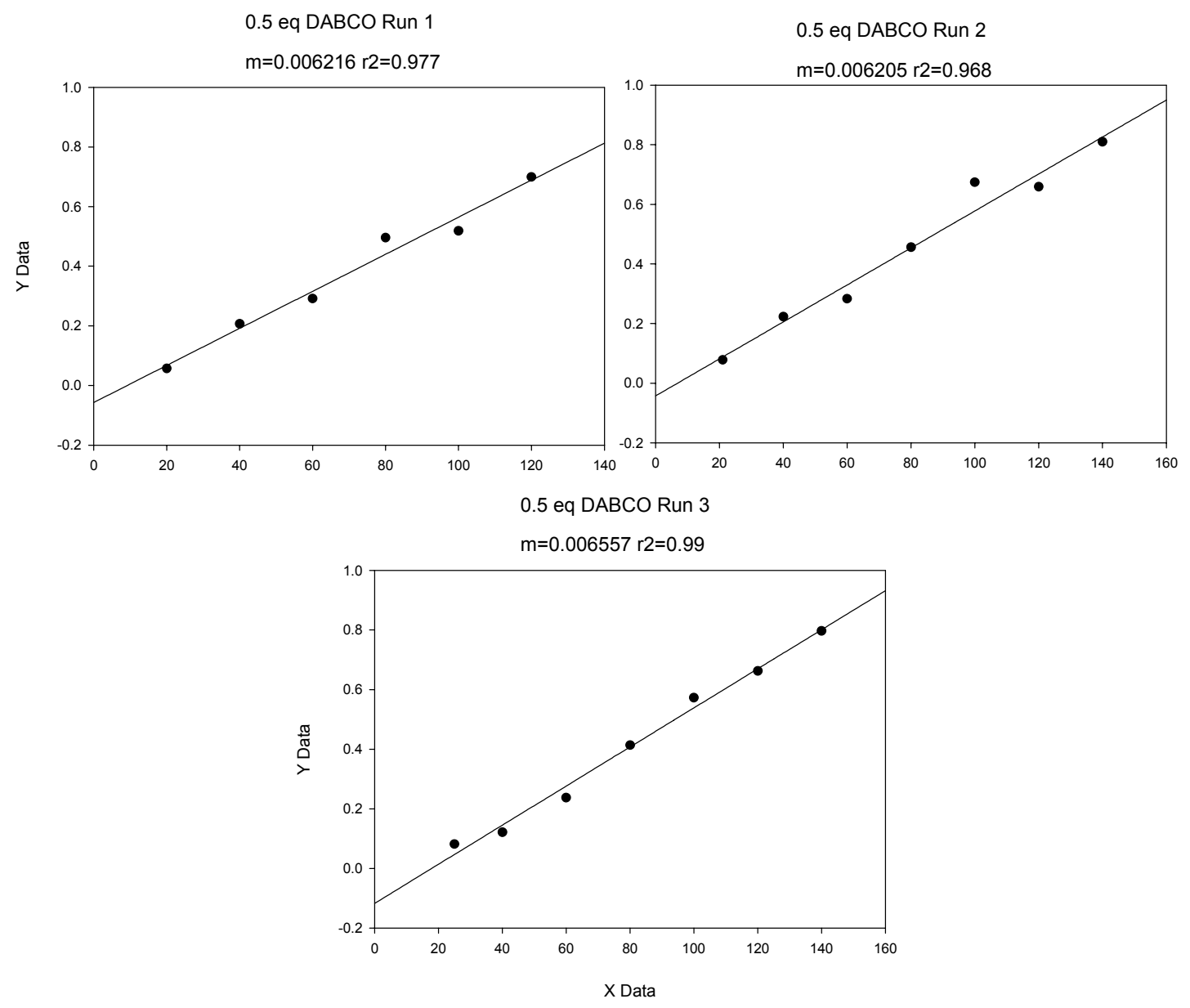


B. Order in 4-nitrobenzaldehyde: The mol of aldehyde + THF were kept constant to keep the DMSO constant throughout. $0.832 \mathrm{M}$ data is taken from the KIE experiments. Please see order plot $\mathrm{F}$ as well where methyl-4-nitrobenzoate was used as the filler rater than THF.

Data Summary:

\begin{tabular}{rrr}
\hline [Aldehyde] & Rate & \multicolumn{1}{c}{ Std Dev } \\
\hline 0.1688 & 0.000192 & $1.788 \mathrm{E}-05$ \\
0.246 & 0.000368 & 0.0000474 \\
0.414 & 0.001122 & 0.000223 \\
0.573 & 0.002057 & 0.0003138 \\
0.832 & 0.00403 & 0.000225 \\
0.333 & 0.0006386 & 0.0001 \\
0 & 0 & 0
\end{tabular}

Order Plot:
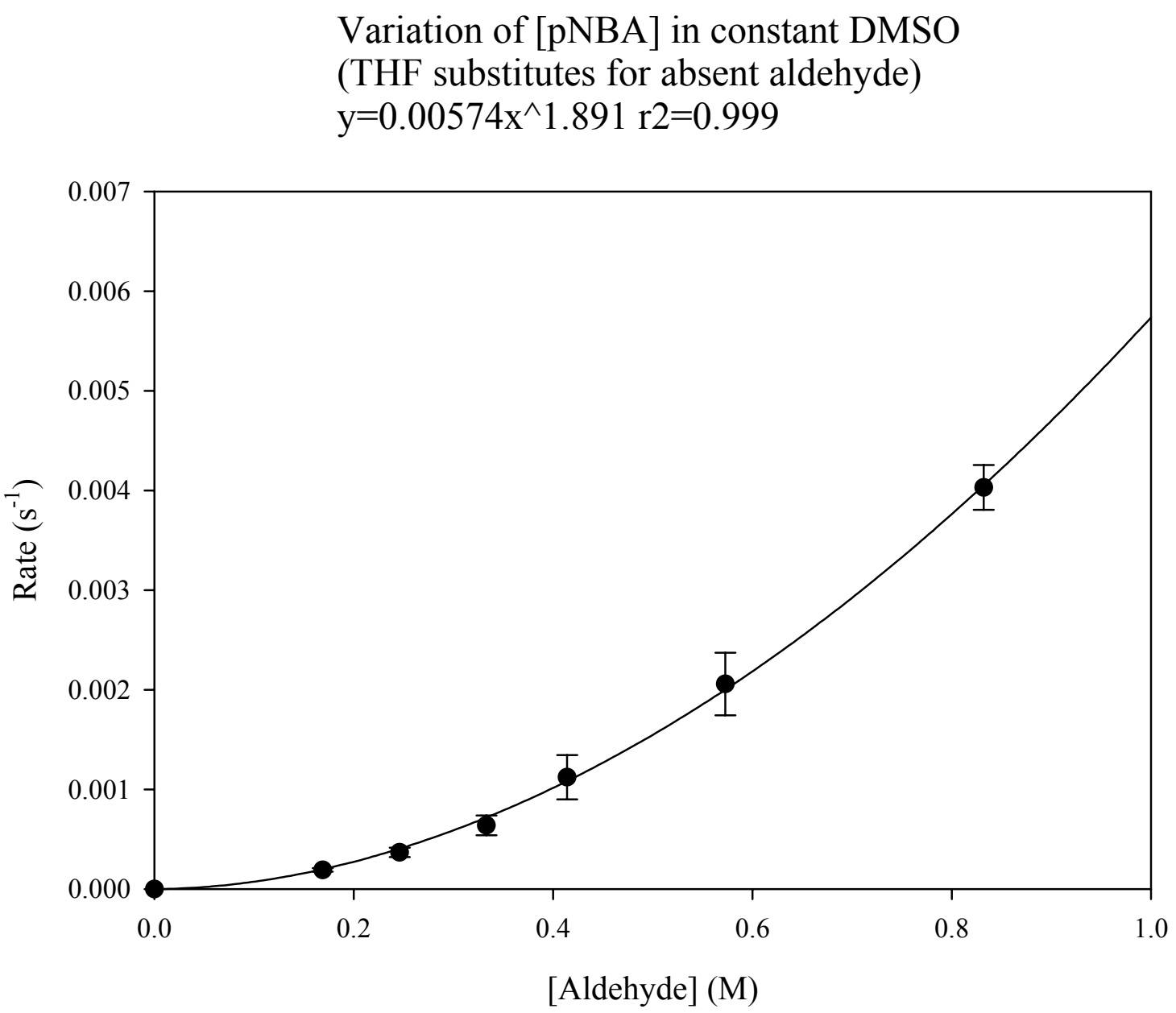
0.2 eq Aldehyde

Average Rate $=0.000192$

Std dev $=0.0000178(9.2 \%)$

0.2 eq Run 2

$\mathrm{m}=0.0002111 \mathrm{r} 2=0.9414$

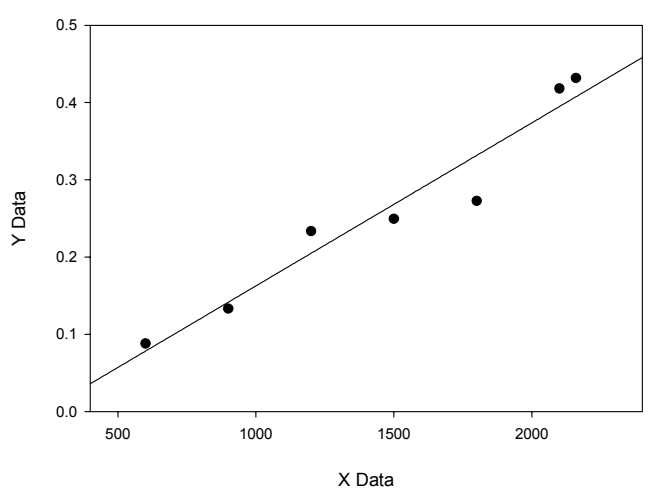

0.2 eq Run 3

$\mathrm{m}=0.0001895 \mathrm{r} 2=0.983$

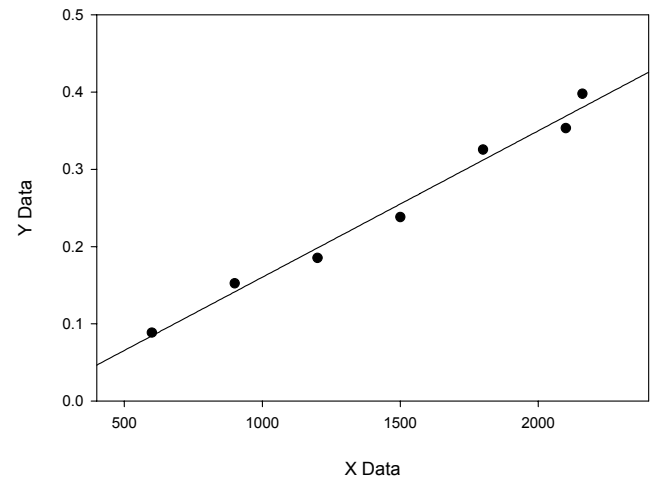

0.2 eq Run 1

$\mathrm{m}=0.0001755 \mathrm{r} 2=0.951$

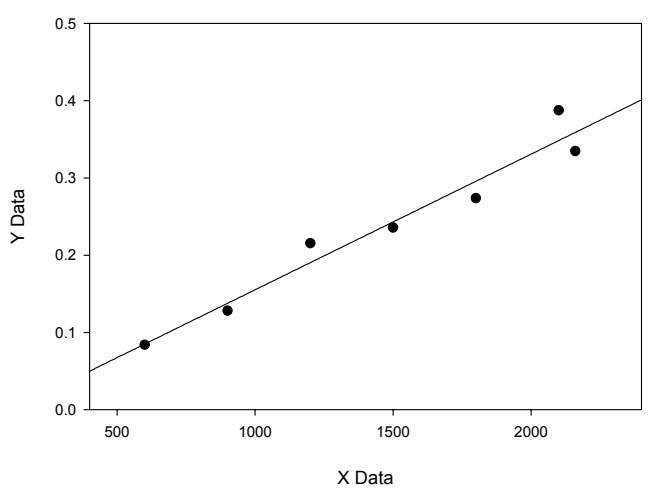


0.3 eq Aldehyde

Average Rate $=3.68 \times 10^{\wedge}-4$

Std dev $=4.75 \times 10^{\wedge}-5(13 \%)$
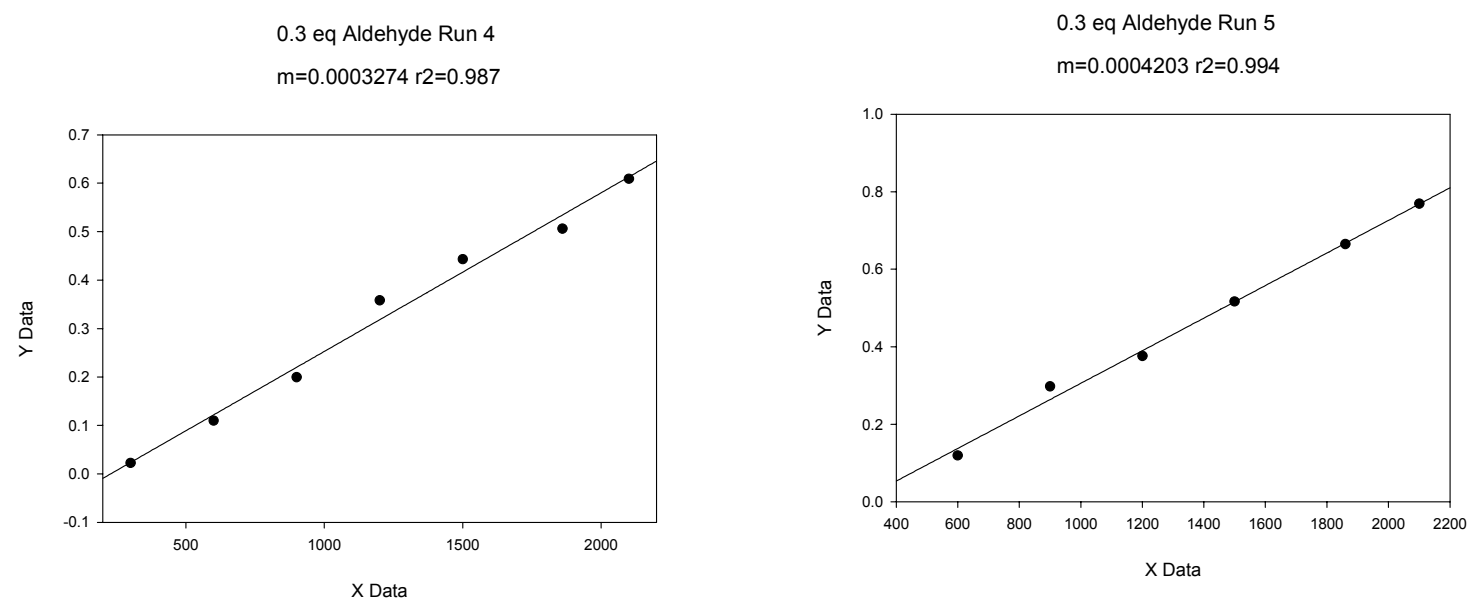

0.3 eq Aldehyde Run 6

$\mathrm{m}=0.0003567 \mathrm{r} 2=0.995$

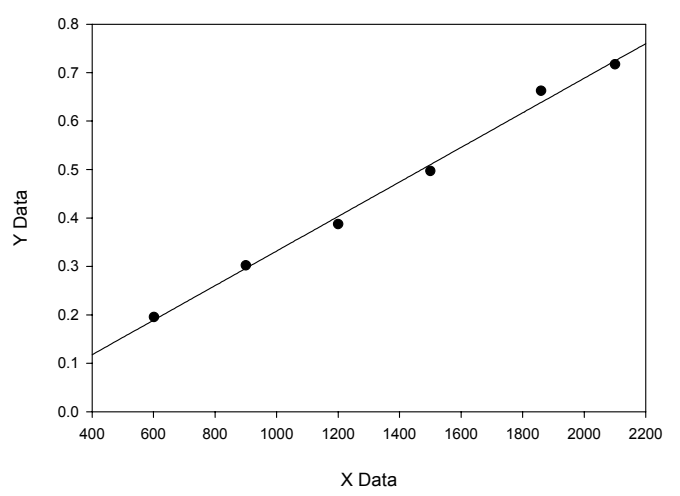


0.5 eq Aldehyde

Average Rate $=0.001122$

Std dev $=0.000223$

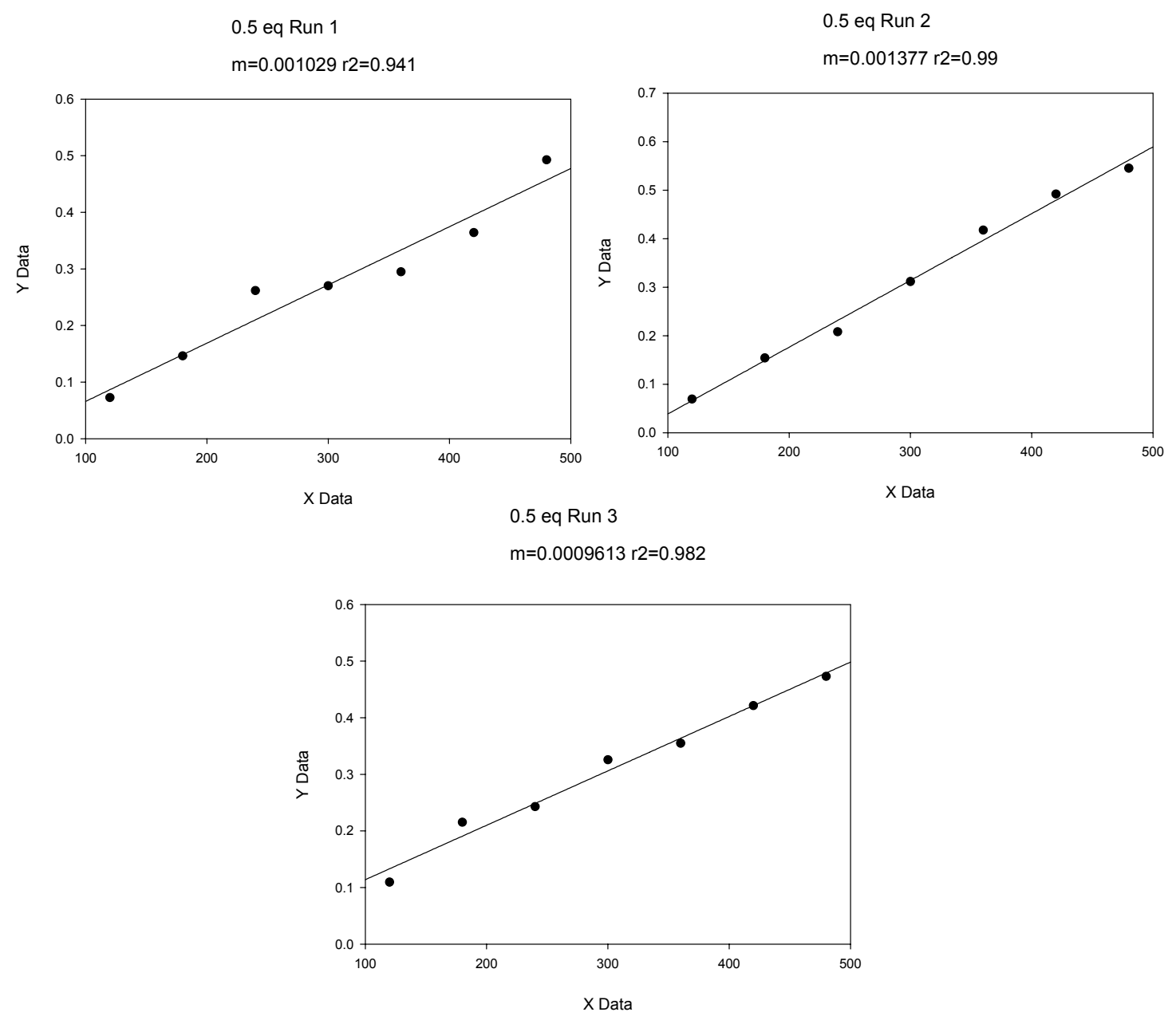


0.7 eq Aldehyde

avg rate $=0.002057$

std dev= 3.138 e-4 (15\%)
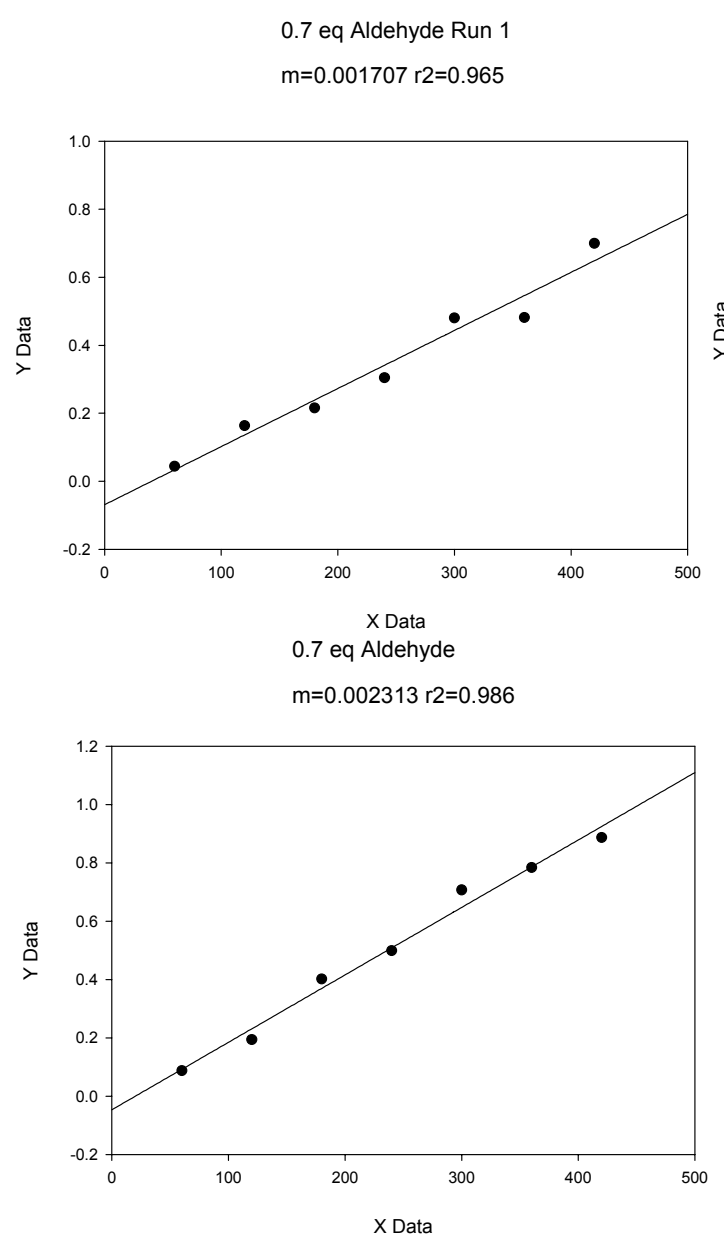

0.7 eq Aldehyde

$\mathrm{m}=0.002152 \mathrm{r} 2=0.989$

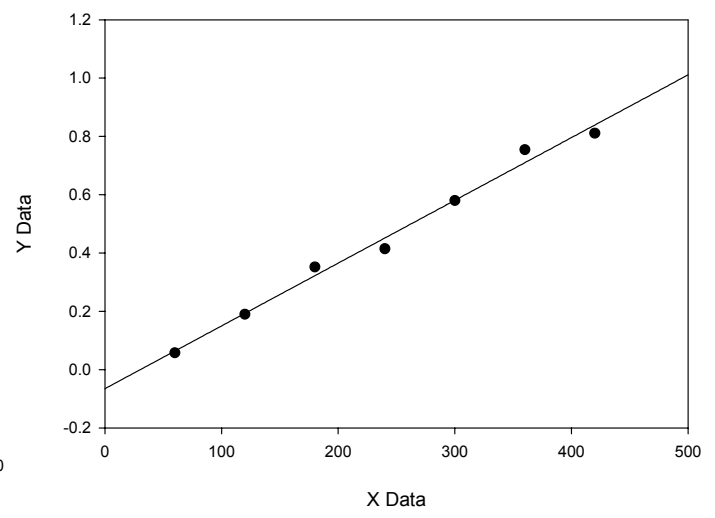


0.4 eq Aldehyde

Average Rate $=6.39 \mathrm{e}-4+/-1 \mathrm{e}-4(15 \%)$
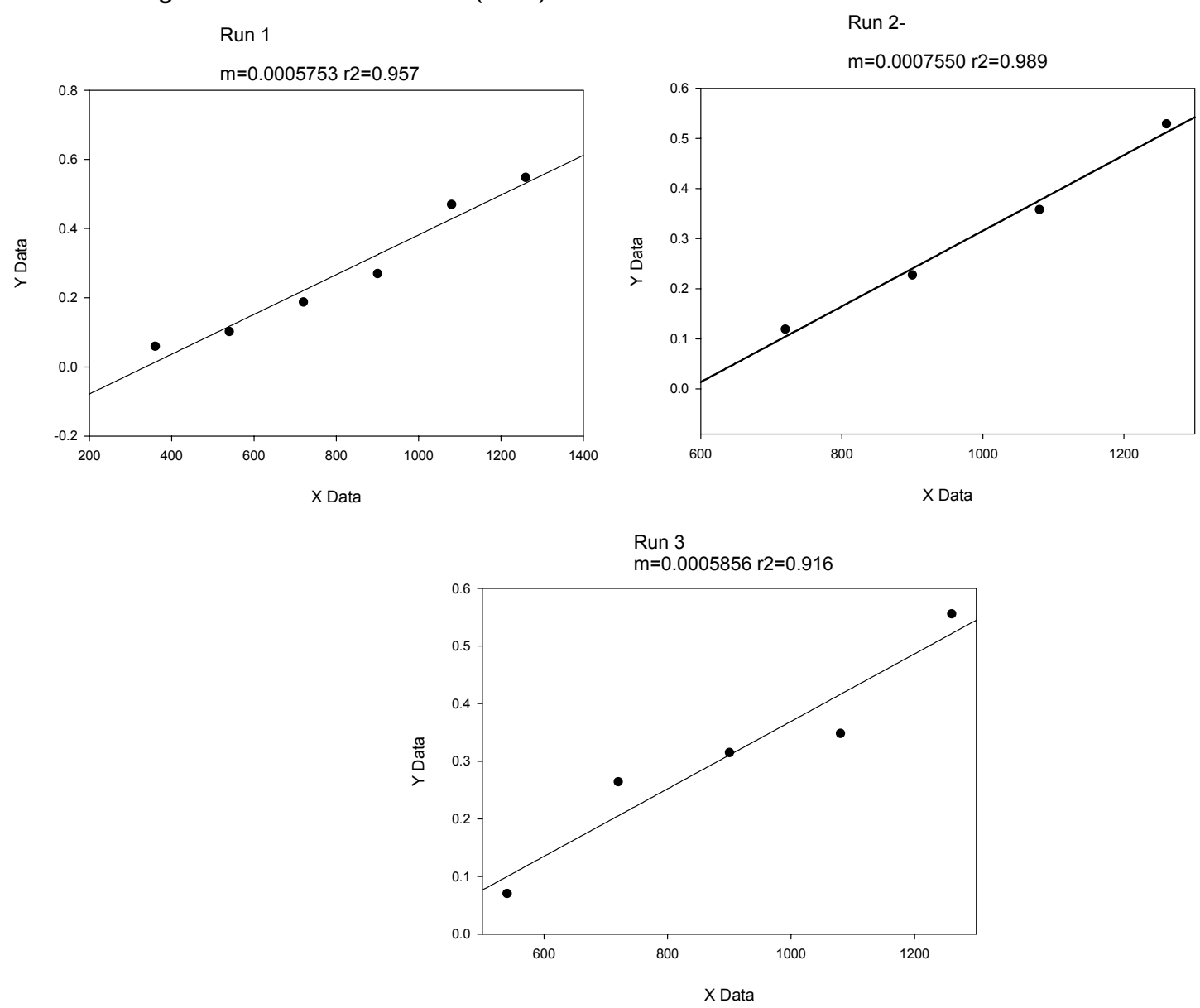
C. Order in Methyl Acrylate: Mol of THF+MA are kept constant to preserve a constant DMSO concentration. $0.84 \mathrm{M}$ point was taken from the KIE data.

Data Summary:

\begin{tabular}{rrr}
\hline [MA] & \multicolumn{1}{c}{ Rate } & \multicolumn{1}{c}{ Std Dev } \\
\hline 0.0831667 & 0.0002778 & $1.899 \mathrm{E}-05$ \\
0.1996 & 0.0008707 & 0.0000408 \\
0.4158333 & 0.00199 & 0.000148 \\
0.6320667 & 0.00266 & 0.000269 \\
0.84 & 0.00403 & 0.000225
\end{tabular}

Order Plot:
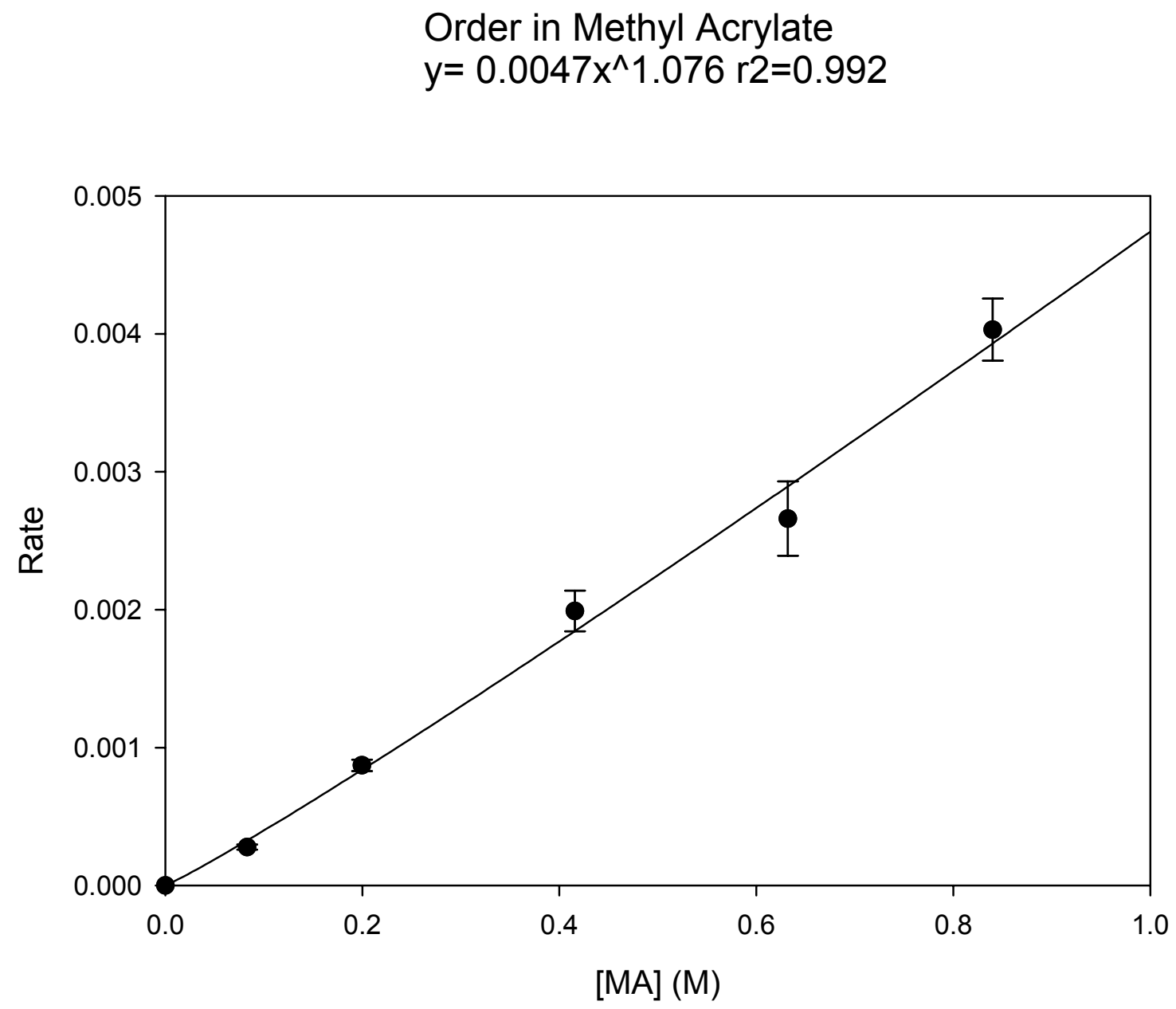
0.1 eq Methyl Acrylate

Average Rate $=0.0002777$ Std dev $=0.0000189(6.8 \%)$

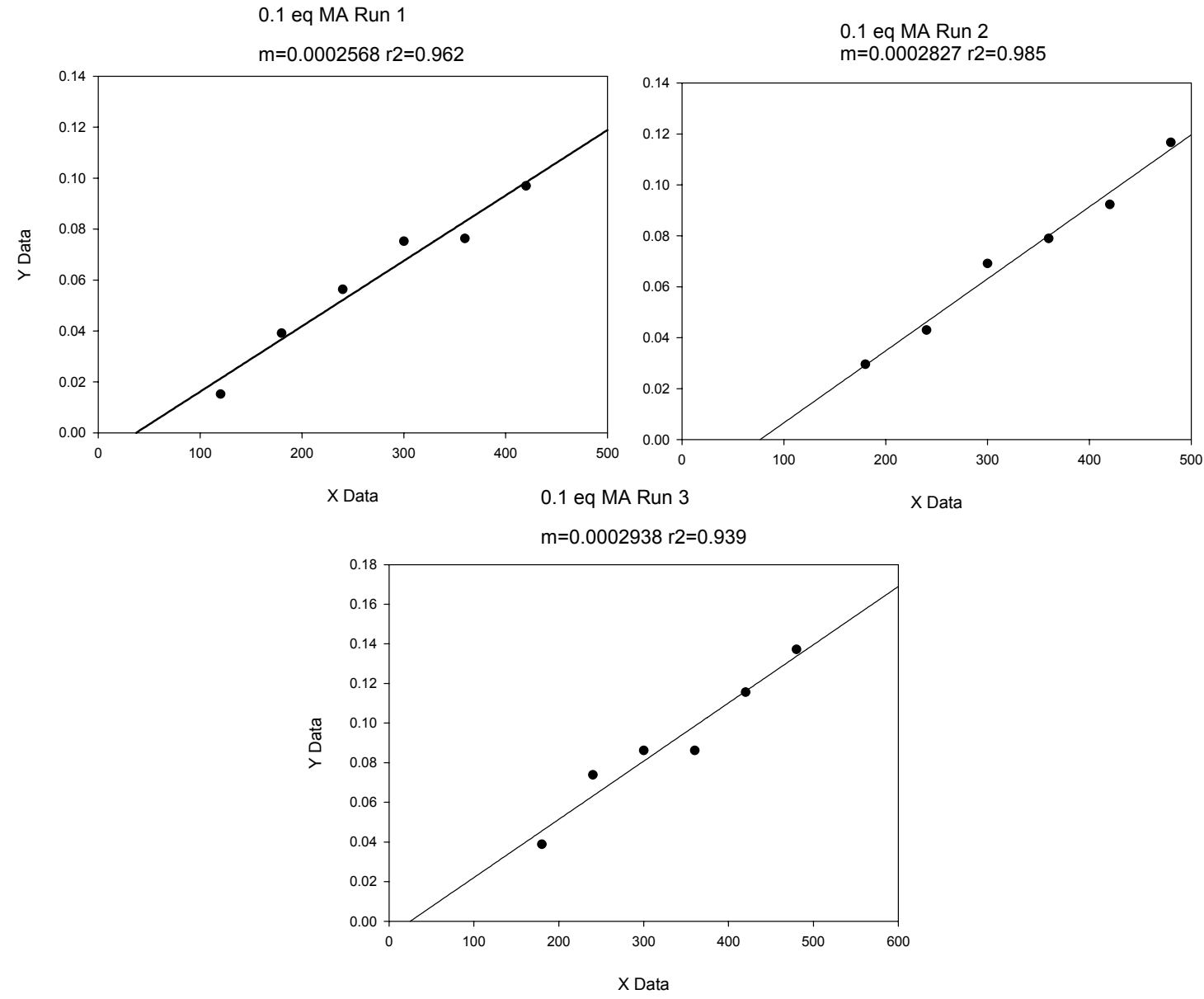


0.25 eq MA

Average Rate $=0.0008707$ Std dev $=0.0000408(4.7 \%)$
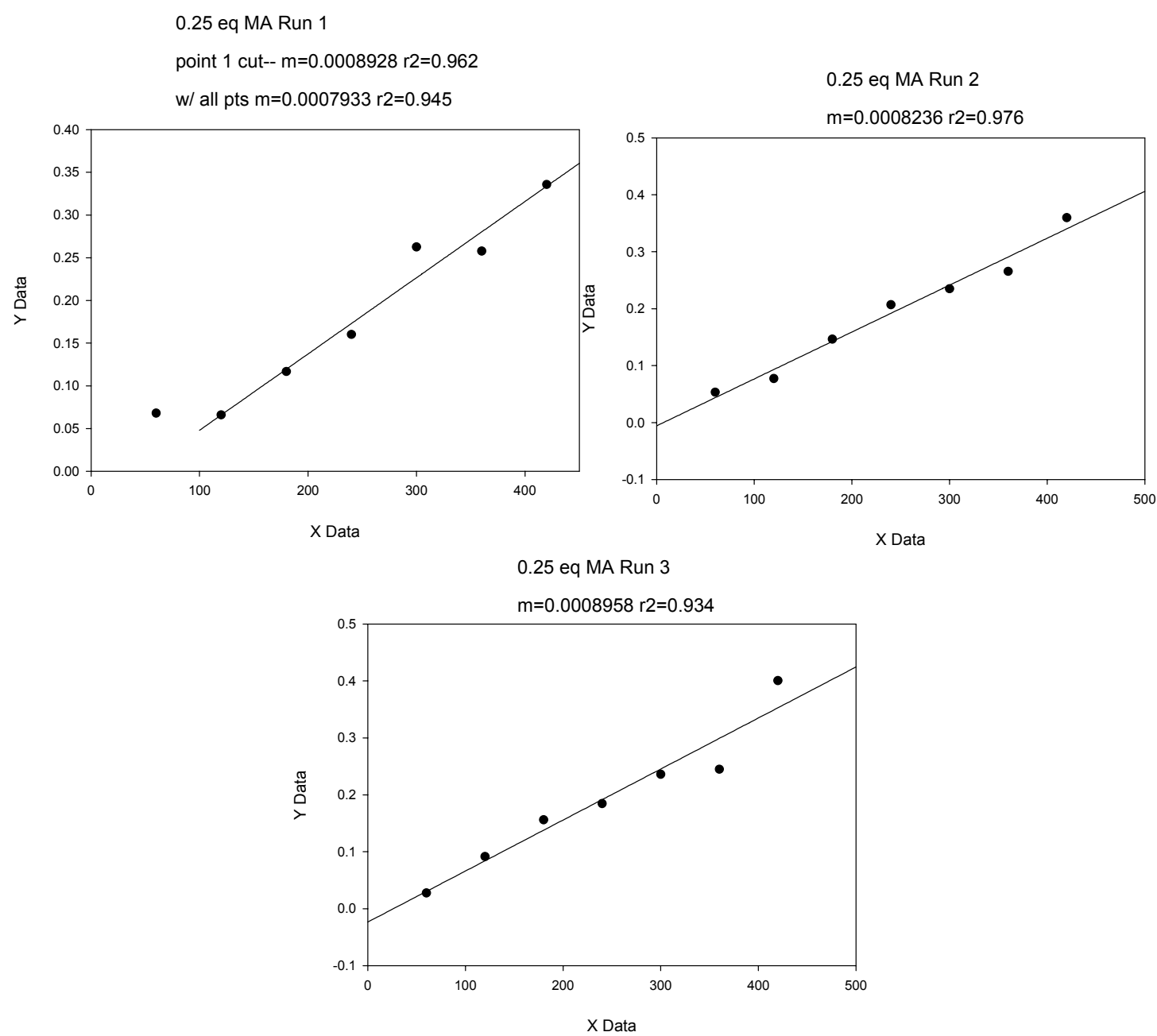
0.5 eq MA--

Average Rate $=0.00199$ Std dev $=0.000148(7.4 \%)$
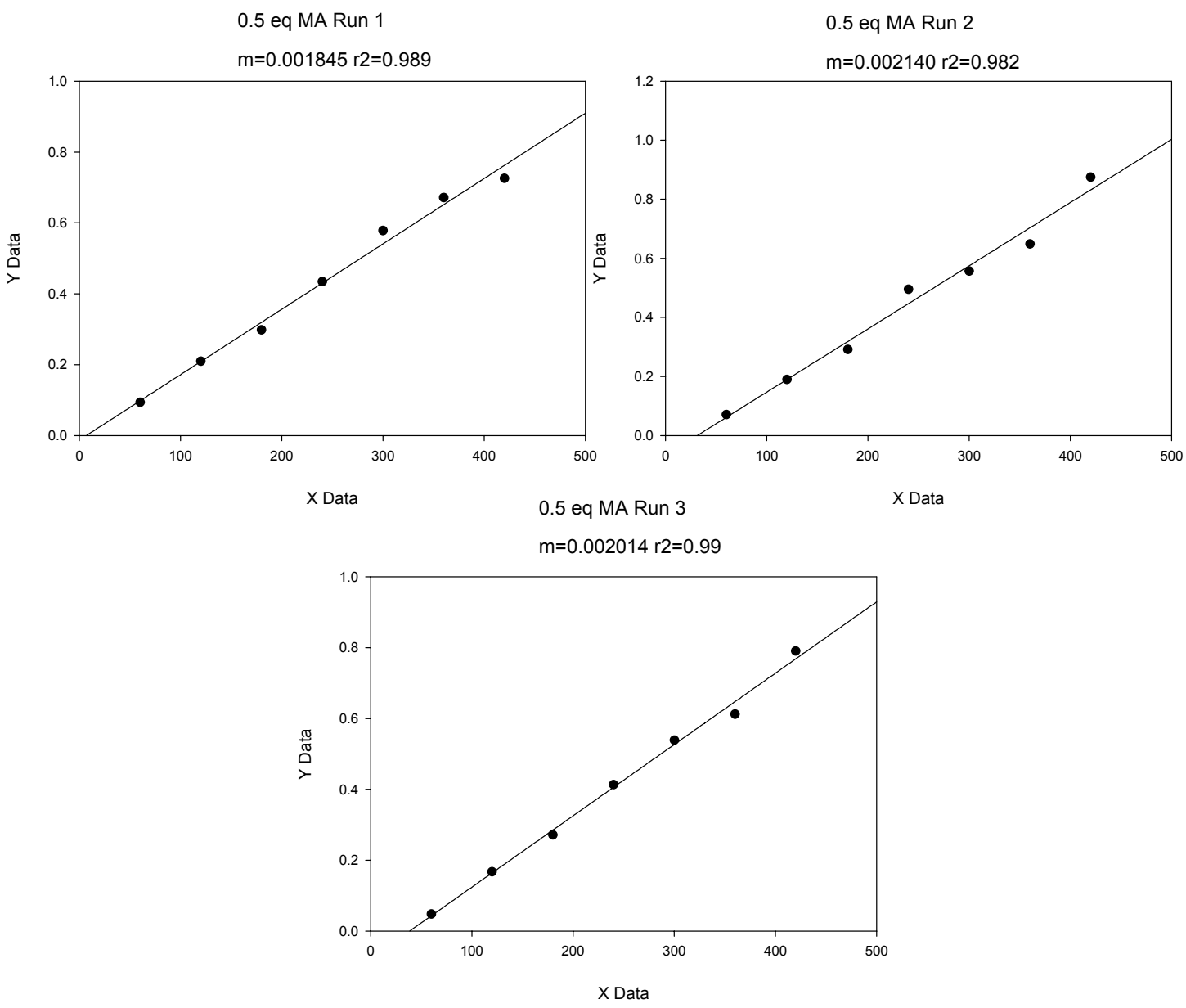
0.75 eq MA Data:

Run $1 \mathrm{t}$-tests out at $95 \%$ confidence level

Average Rate- 0.00266125 std dev $=0.00026988$
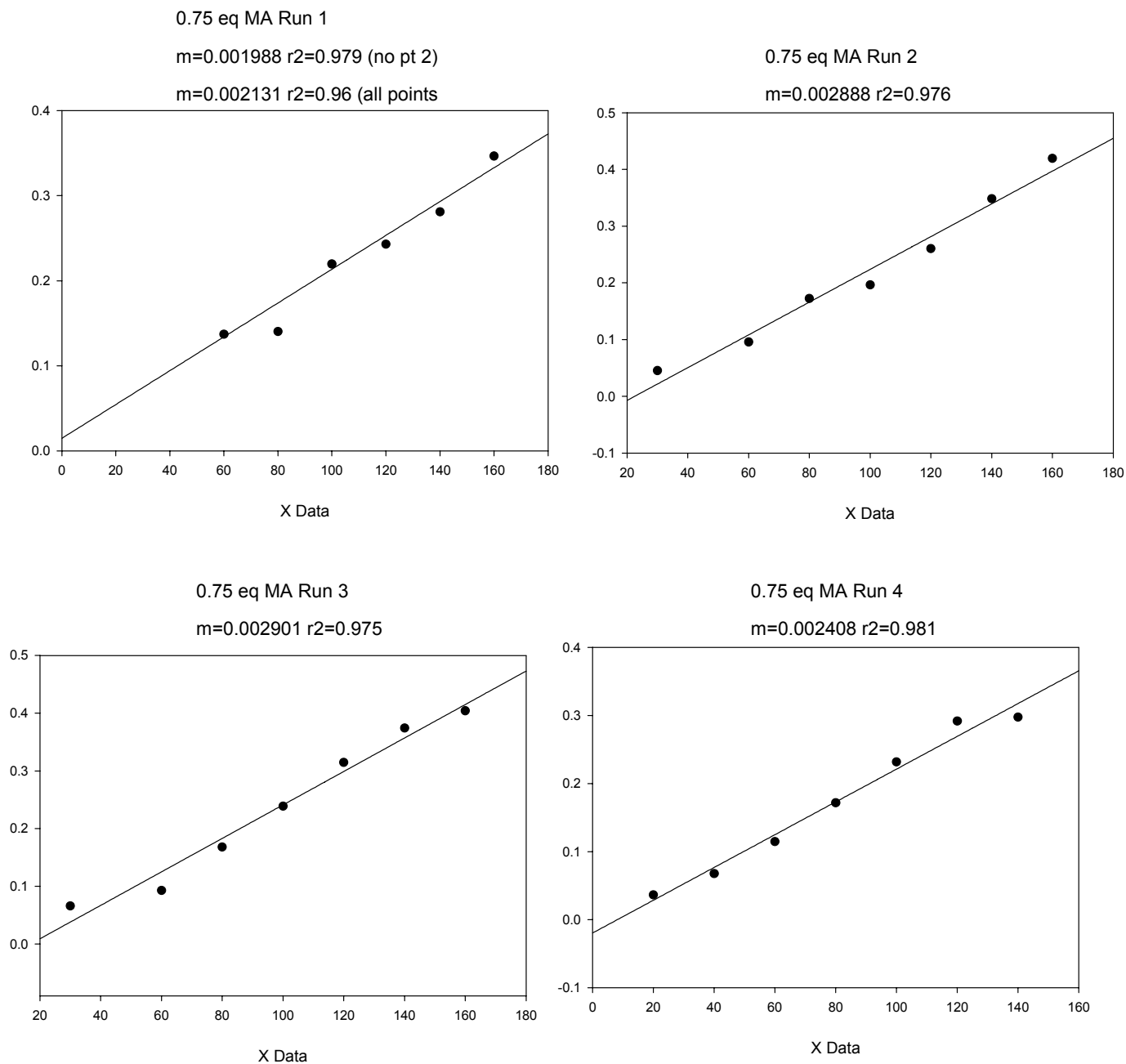

0.75 eq MA Run 5

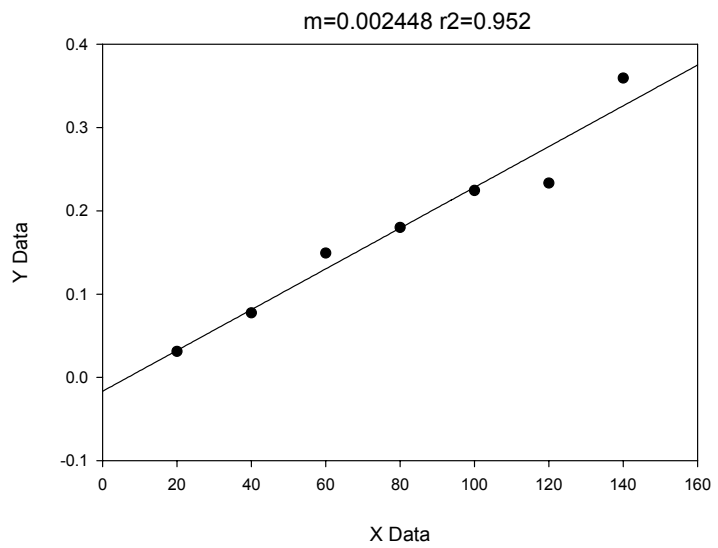




\section{Order in DMSO in Chloroform}

Data Summary:

\begin{tabular}{rrr}
\hline [DMSO] & \multicolumn{1}{l}{ Rate } & \multicolumn{1}{l}{ Std Dev } \\
\hline 8 & 0.00231775 & 0.000277477 \\
6 & 0.001863667 & 0.000180359 \\
4 & 0.001149 & 0.000190596 \\
0 & 0.000114 & 0.00000834 \\
14 & 0.00403 & 0.000225
\end{tabular}

Order Plot:

Order in DMSO in Chloroform $y=a x^{\wedge} b+c a=0.0002725 b=0.996 c=0.00005878$

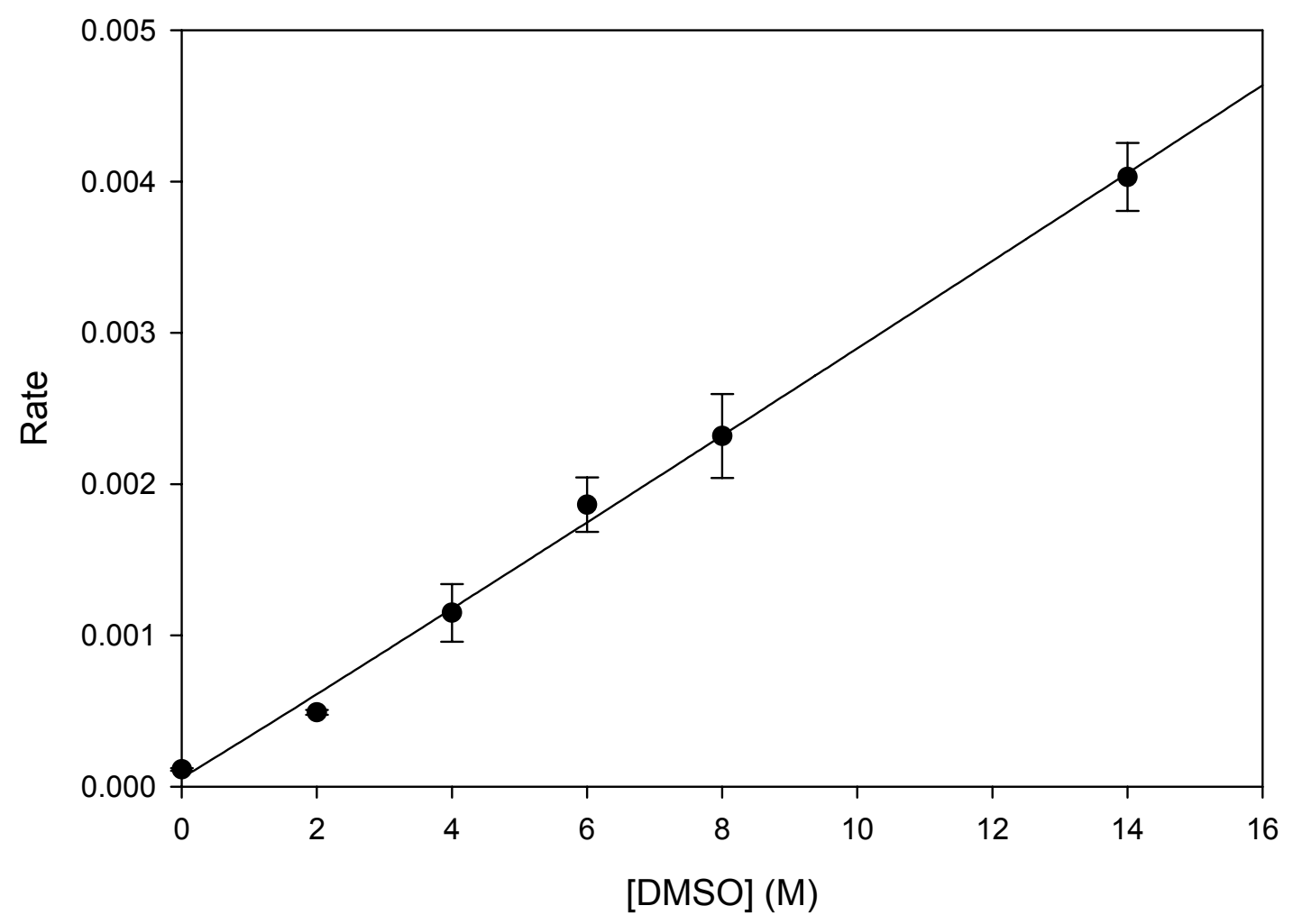




\section{Initial Rate Plots:}

8M DMSO Run 1

$\mathrm{m}=0.001950 \mathrm{r} 2=0.9914$

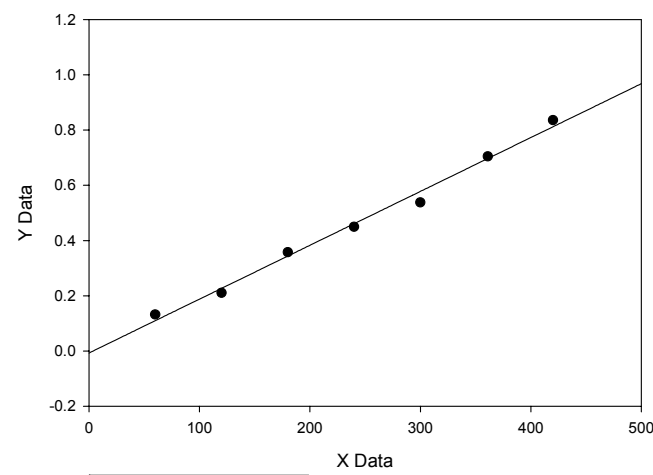

$$
\text { - Time 8M vs 8M Run } 1
$$

8 M DMSO Run 3

$\mathrm{m}=0.002354 \mathrm{r} 2=0.9899$

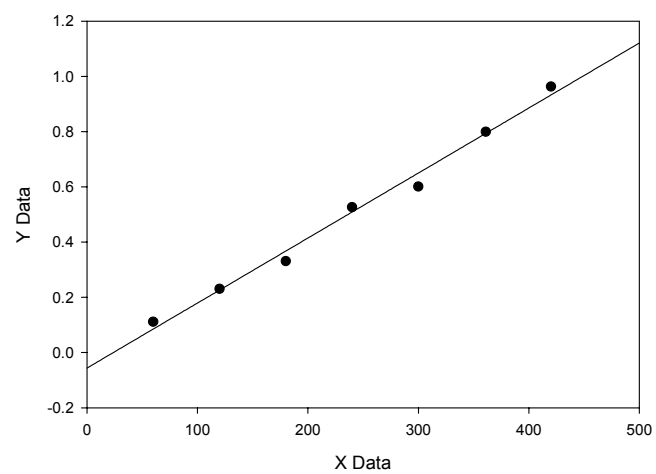

$$
\begin{aligned}
& \text { - Time 8M vs } 8 \mathrm{M} \text { Run } 3 \\
& \mathrm{x} \text { column } 2 \text { vs y column } 2
\end{aligned}
$$

6 M DMSO Run 1 $\mathrm{m}=0.001801 \mathrm{r} 2=0.992$

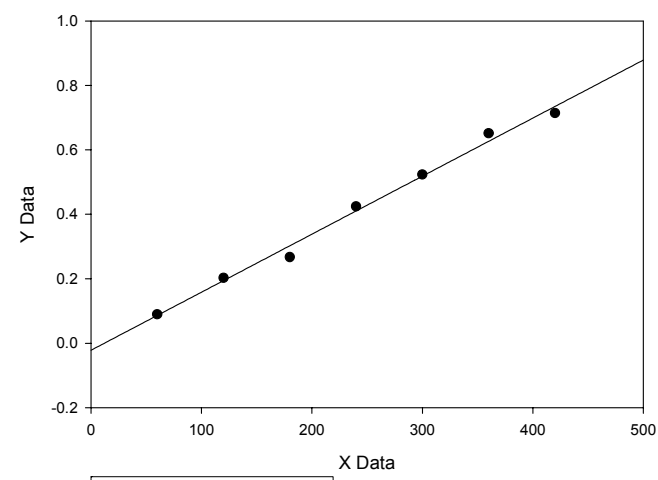

- Time 6 M vs 6 M Run 1
x column 3 vs y column 3
8 M DMSO Run 2

$\mathrm{m}=0.002343 \mathrm{r} 2=0.976$

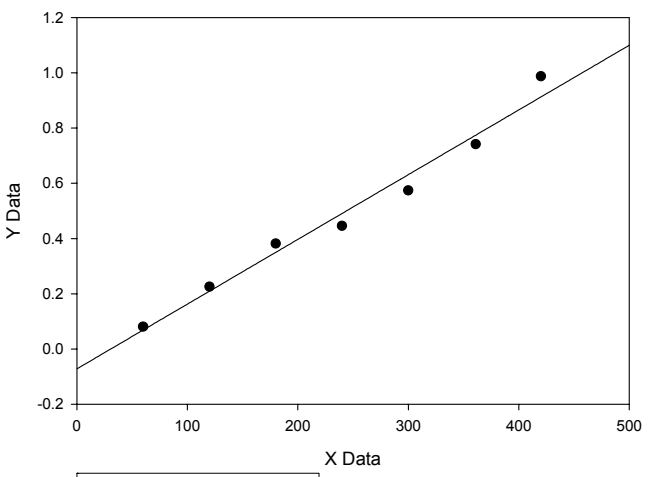

- Time 8M vs 8M Run 2

8M DMSO Run 4

$\mathrm{m}=0.002624 \mathrm{r} 2=0.994$

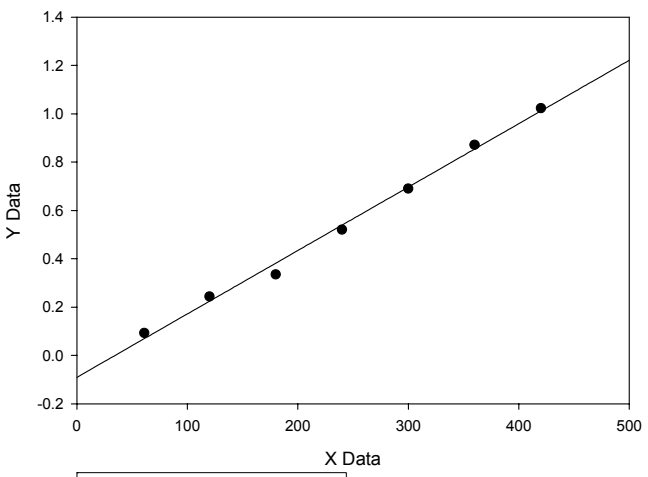

- Time 8M Run 4 vs 8 M Run 4 - $x$ column 6 vs y column 6

6 M DMSO Run 2 $\mathrm{m}=0.001723 \mathrm{r} 2=0.979$

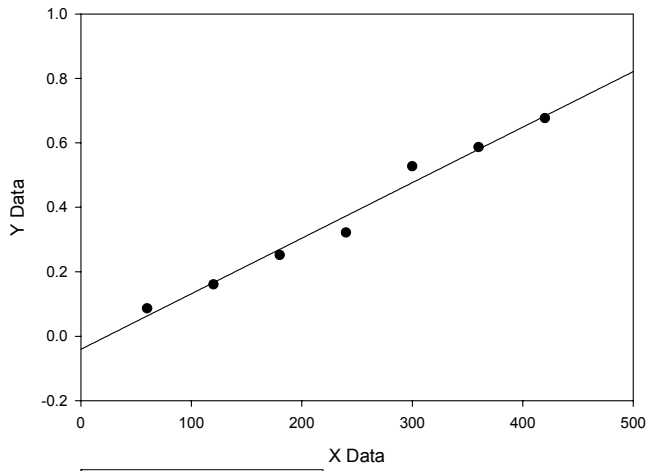

- Time $6 \mathrm{M}$ vs $6 \mathrm{M}$ Run 2 

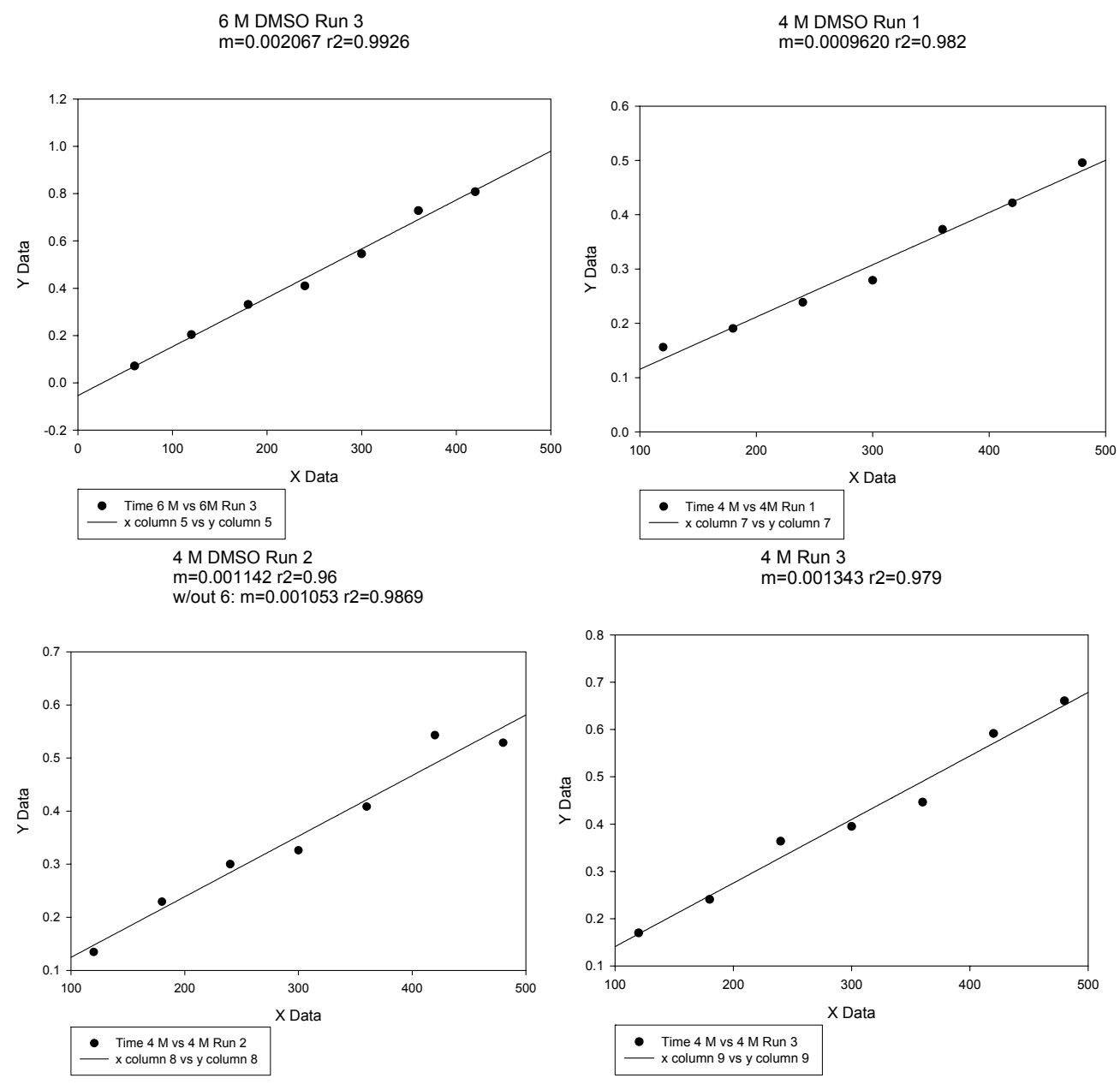
2 M DMSO Run 1

$\mathrm{m}=0.0004861 \mathrm{r} 2=0.984$

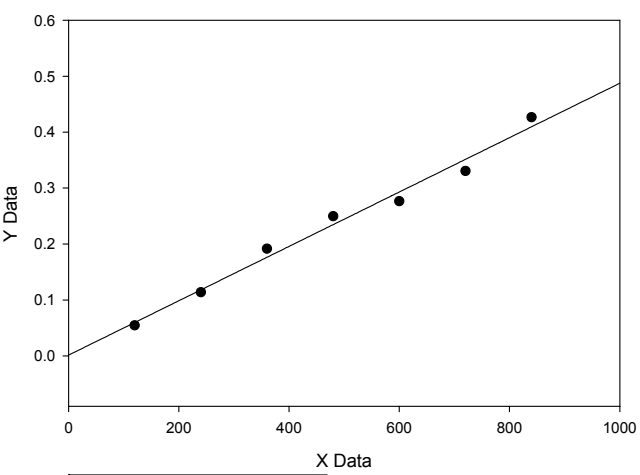

- Time $2 \mathrm{M}$ vs $2 \mathrm{M}$ Run 1

2M DMSO Run 3

$\mathrm{m}=0.0004774 \mathrm{r} 2=0.957$

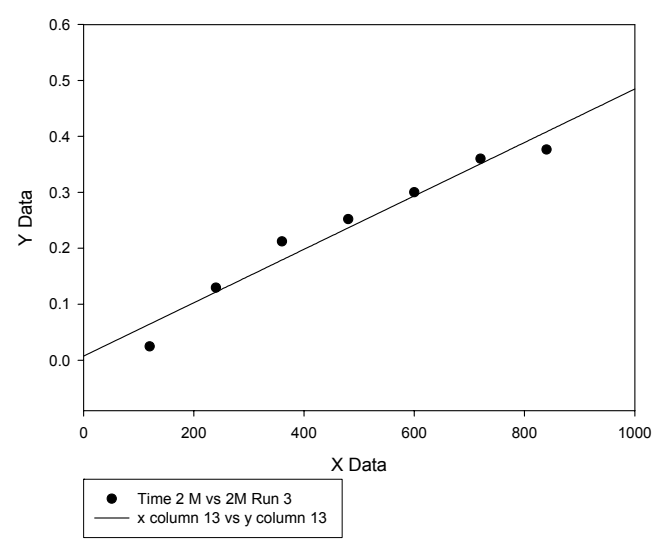

2M DMSO Run 2

$\mathrm{m}=0.0005087 \mathrm{r} 2=0.96$

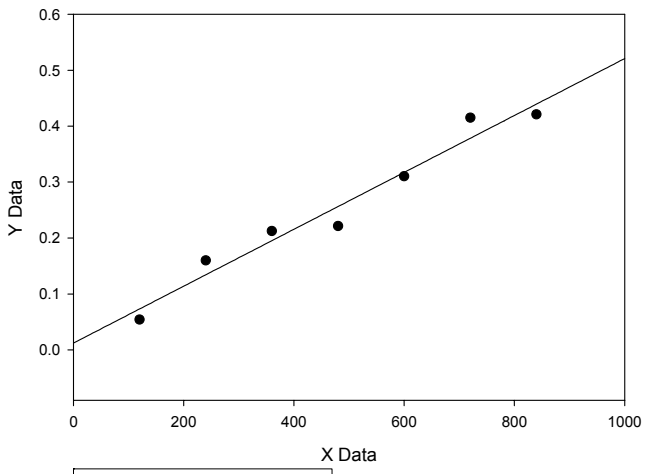

- Time $2 \mathrm{M}$ vs $2 \mathrm{M}$ Run 2
$\mathrm{x}$ column 12 vs y column 1 


\section{E. Order in DMSO in DMF}

Data Summary:

\begin{tabular}{rrr}
\hline [DMSO] & \multicolumn{1}{l}{ Rate } & \multicolumn{1}{l}{ Std Dev } \\
\hline 0 & 0.001125 & $4.38435 \mathrm{E}-05$ \\
2 & 0.0015687 & 0.000216588 \\
4 & 0.002008 & $7.20625 \mathrm{E}-05$ \\
6 & 0.0023837 & 0.000100898 \\
8 & 0.0033673 & 0.000158039 \\
14 & 0.00403 & 0.000225
\end{tabular}

Order Plot:

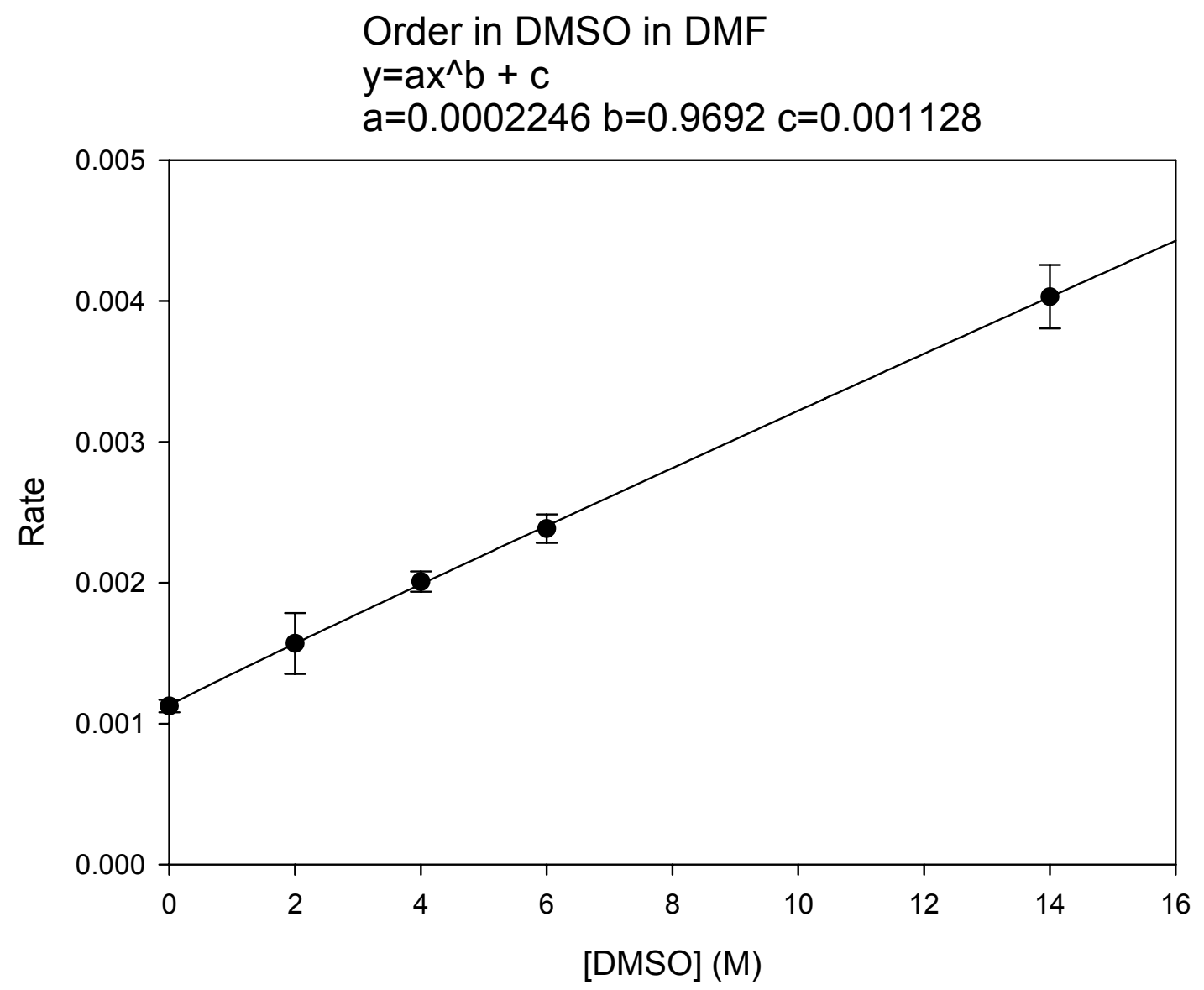




\section{Initial Rate Plots:}

2M DMSO Run 1

$\mathrm{m}=0.001614 \mathrm{r} 2=0.978$

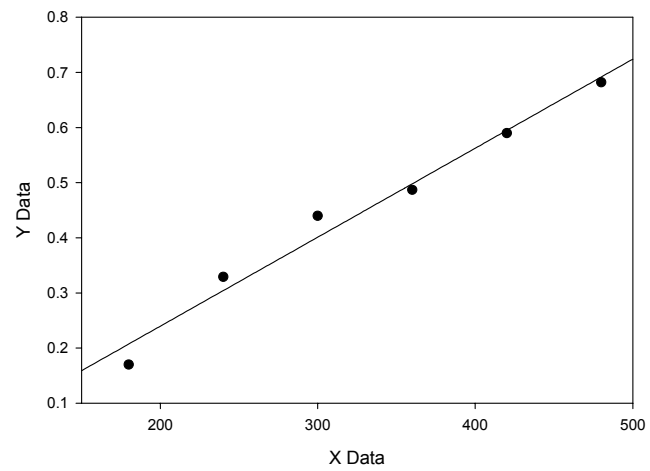

- Time 2M vs 2M DMSO Run 1 - $x$ column 7 vs y column 7

2M DMSO Run 3 $\mathrm{m}=0.001333 \mathrm{r} 2=0.962$
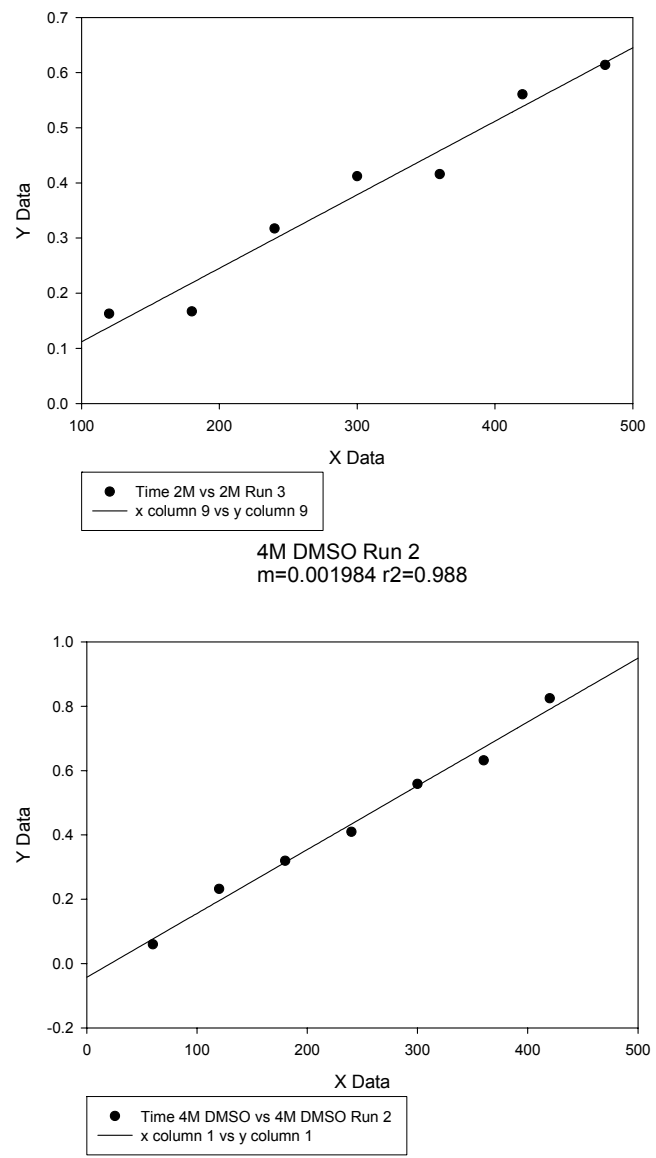

2M DMSO Run 2

$\mathrm{m}=0.001759 \mathrm{r} 2=0.987$
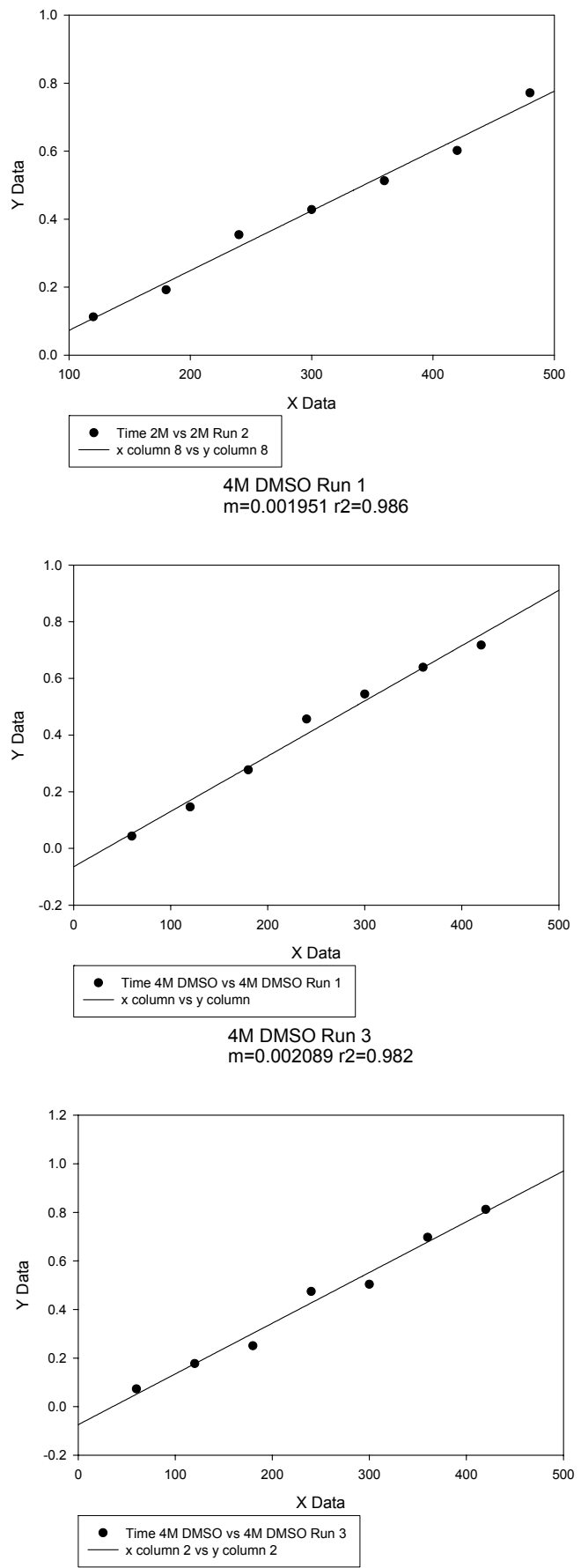

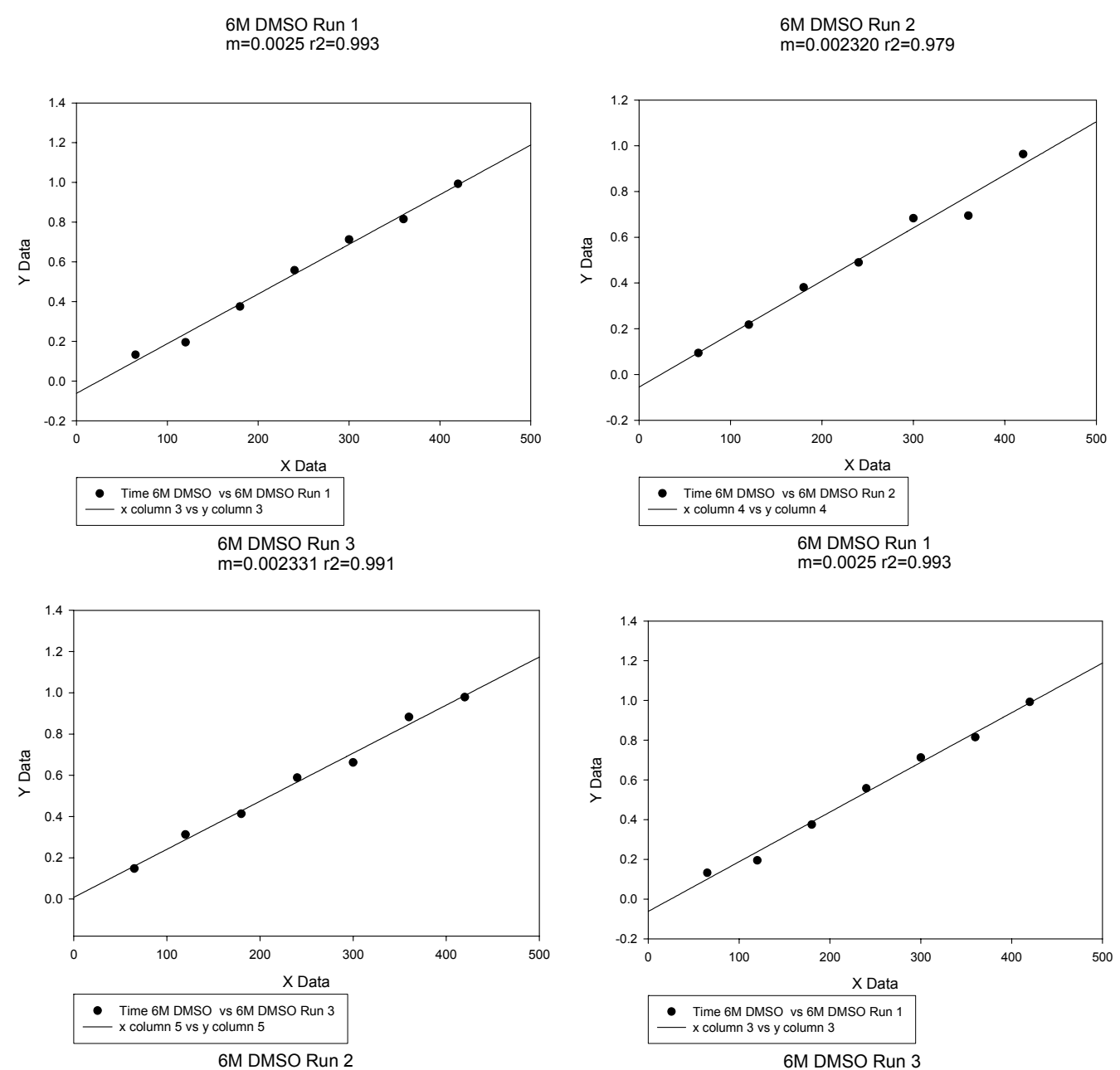

$\mathrm{m}=0.002320 \mathrm{r} 2=0.979$
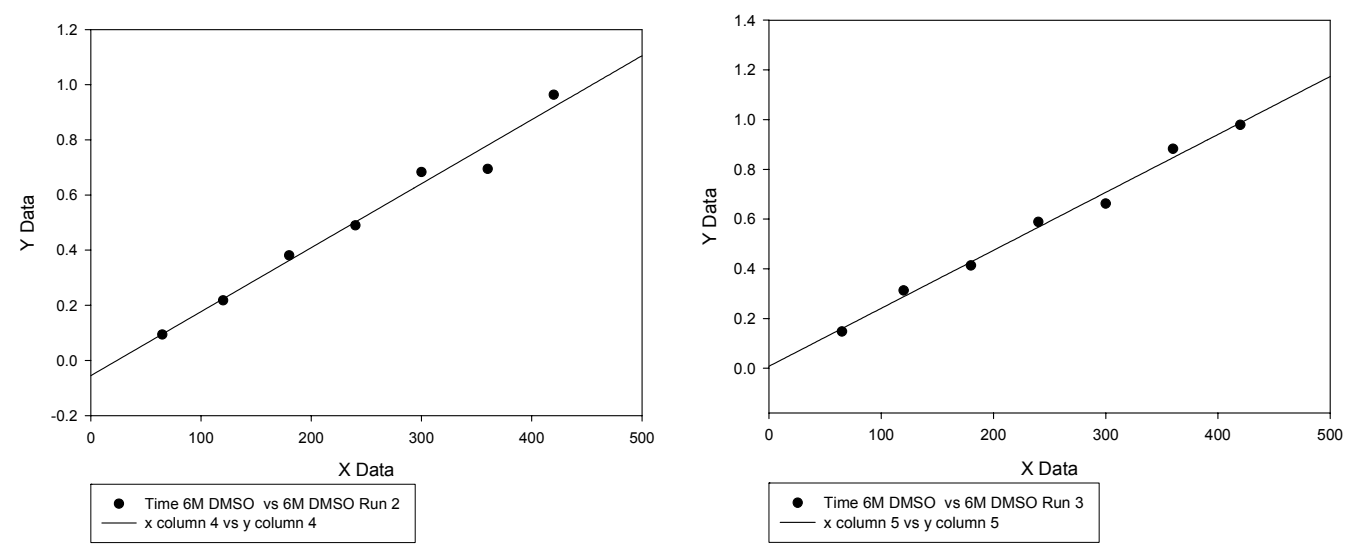
F. Order in p-Nitrobenzaldehyde with Methyl-p-Nitrobenzoate as a filler: As a confirmation of the constant DMSO experiment with THF filler, the absent aldehyde was replaced with the ester. The reaction was run under typical kinetics conditions with constant DMSO. The $0.832 \mathrm{M}$ data was taken from the kinetic isotope effect experiment.

Data Summary:

\begin{tabular}{rrr}
\hline [Aldehyde] & \multicolumn{1}{l}{ Rate } & \multicolumn{1}{c}{ Std Dev } \\
\hline 0.25 & 0.000348 & 0.0000582 \\
0.415 & 0.0010106 & 0.0000446 \\
0.578 & 0.001759 & 0.000193 \\
0.832 & 0.00403 & 0.000225 \\
0 & 0 & 0
\end{tabular}

Initial Aldehyde Rate Plot-DMSO Constant Concentration--- Ester is the place holder for the aldehyde $y=0.005904 x^{\wedge} 2.109 \mathrm{r} 2=0.998$

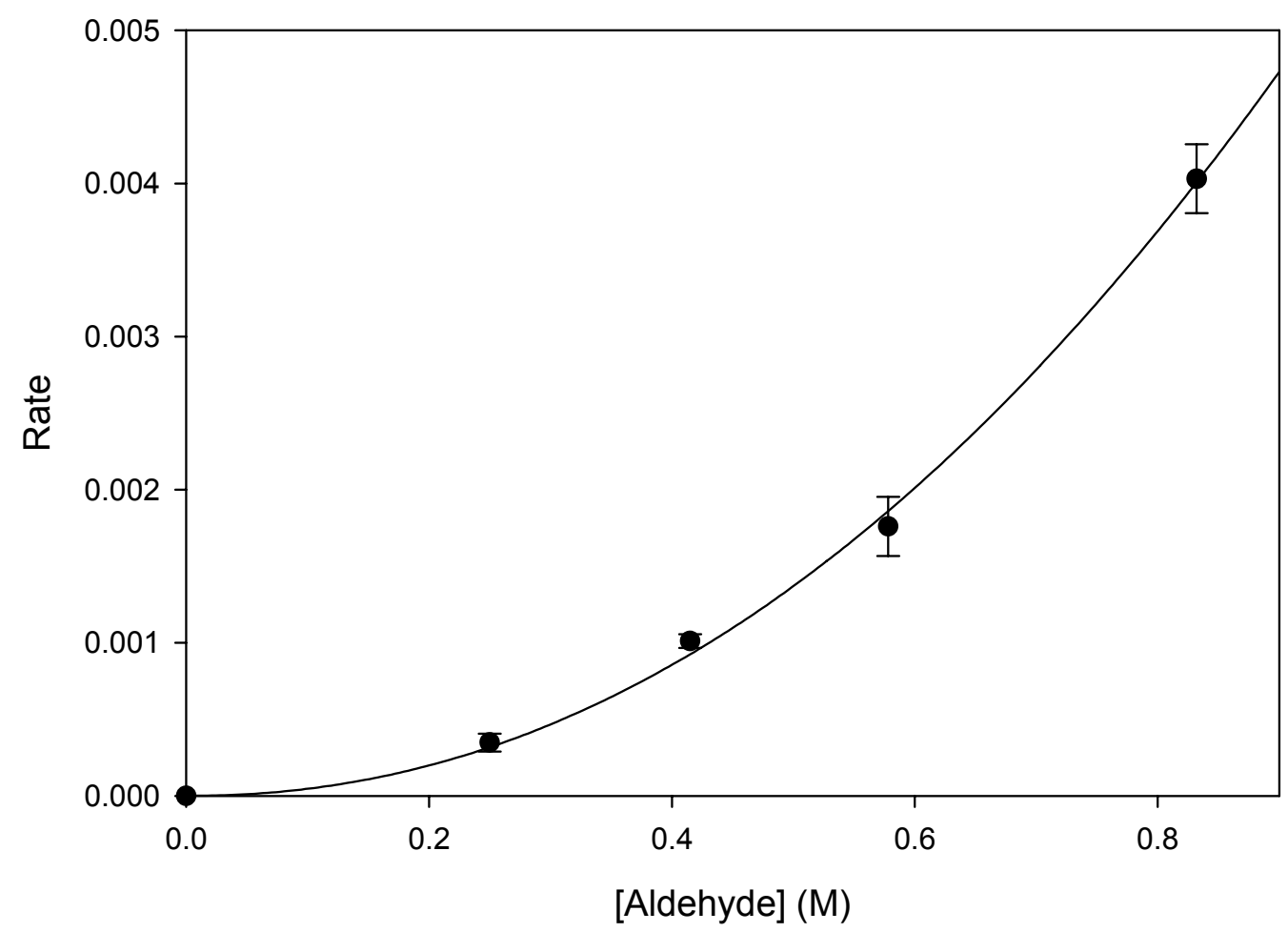


Initial Rate Data:

0.7 eq Aldehyde with the ester

Rate $=0.001759$

Std Dev $=0.000193$ (10.9\% error)

0.7 eq Aldehyde Run 1
$\mathrm{~m}=0.001622 \mathrm{r} 2=0.976$

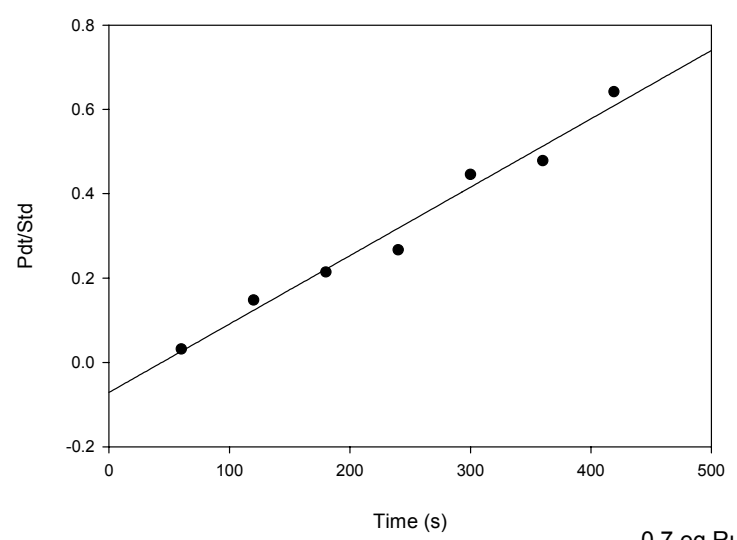

0.7 eq Aldehyde Run 2

$\mathrm{m}=0.001673 \mathrm{r} 2=0.989$

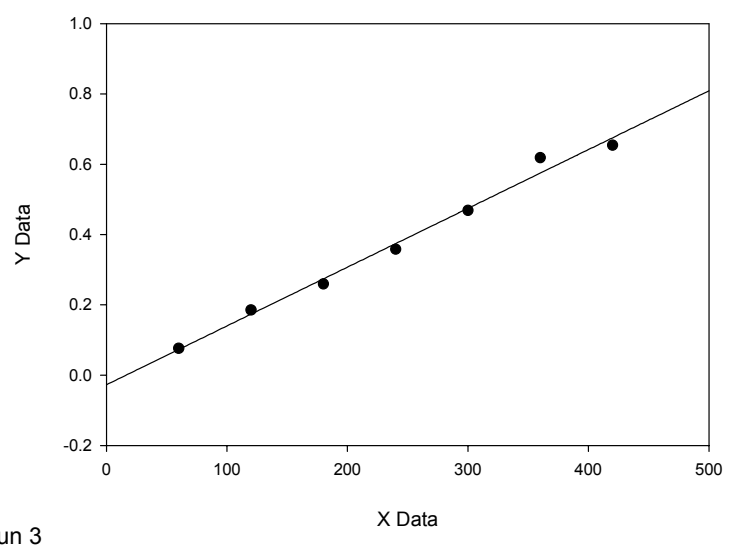

0.7 eq Run 3

$\mathrm{m}=0.001979 \mathrm{r} 2=094$

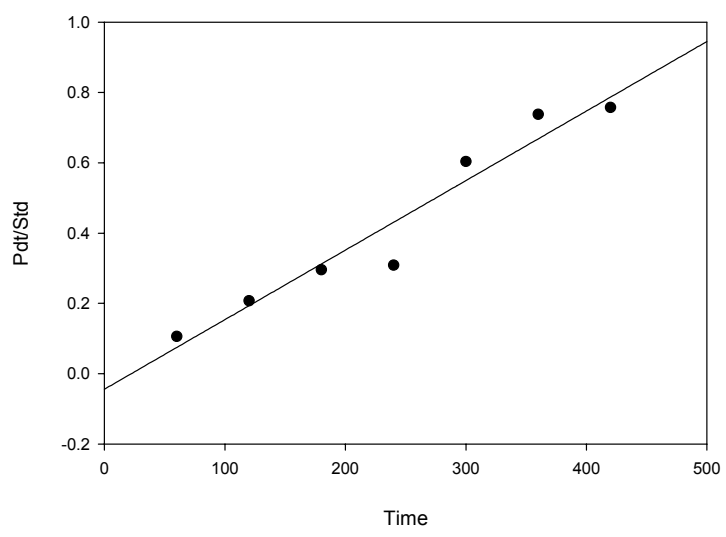




\section{5 eq Aldehyde + methyl-4-nitrobenzoate in DMSO}

Average Rate $=0.0010106$

Std Dev= 0.0000446 (4.4\% error)

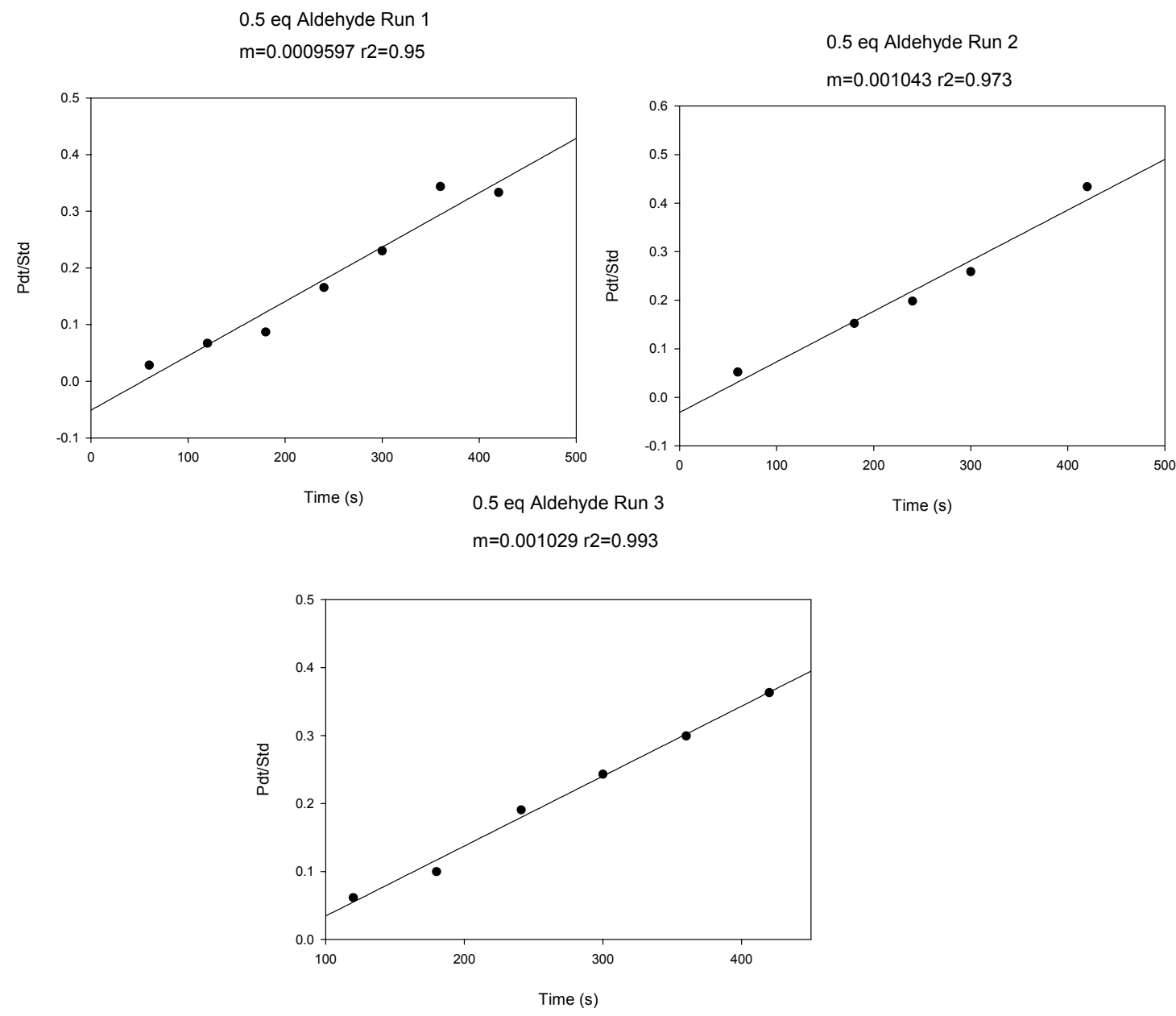




\section{3 eq Aldehyde with methyl-4-nitrobenzoate in DMSO}

Average Rate: $3.4797 \times 10^{\wedge}-4$

Std Dev: 0.0000582 (16.7\% error! --- huge!)

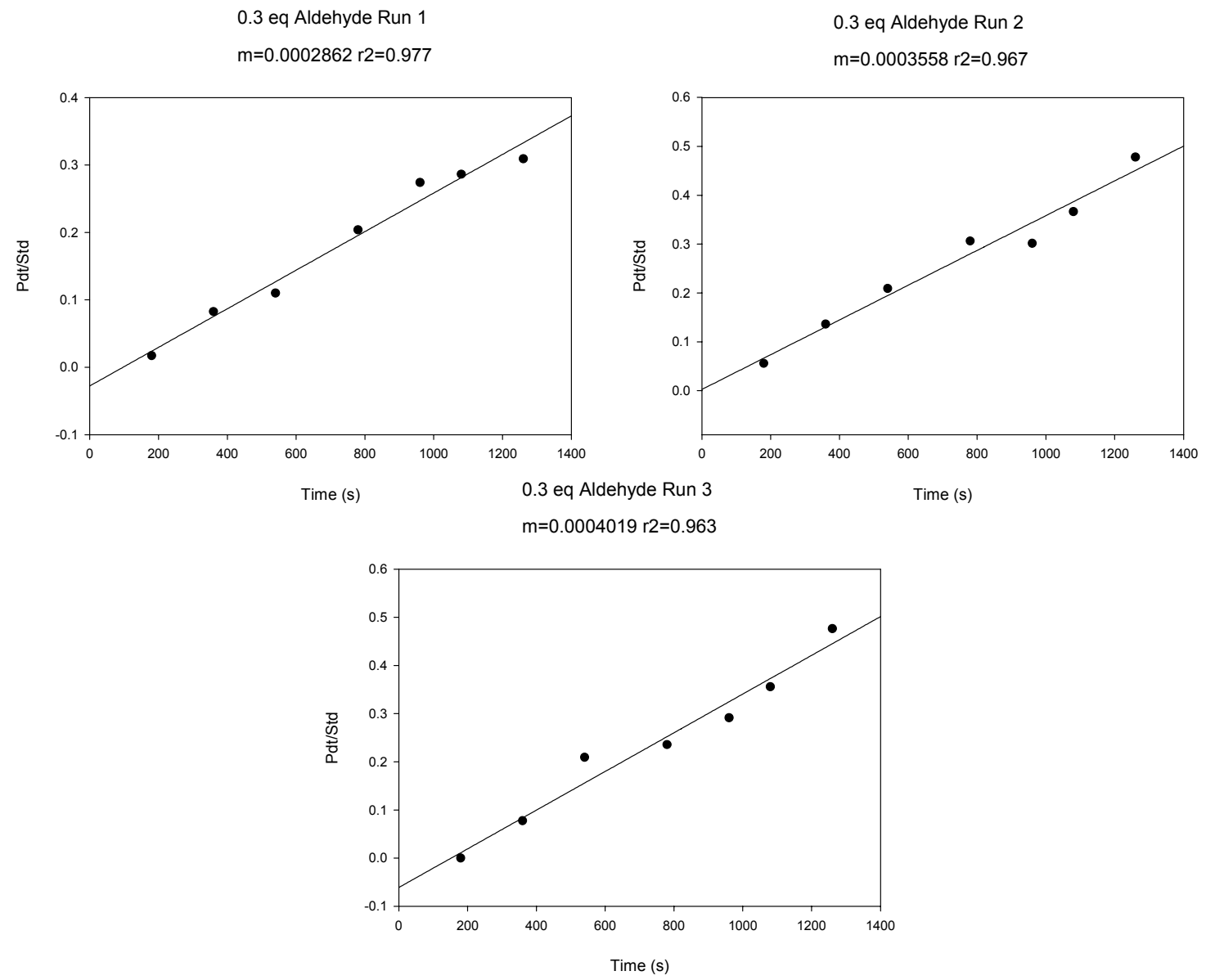




\section{Kinetic Isotope Effect Experiments}

A. 4-Nitrobenzaldehyde in DMSO

$\mathrm{K}_{\mathrm{H}}=0.0040283 \pm 0.0002252(5.6 \%$ error $)$

$\mathrm{K}_{\mathrm{D}}=0.000776 \pm 0.000073$ ( $9.4 \%$ error $)$

$\mathrm{KIE}=5.19 \pm 0.58$

DMSO H1

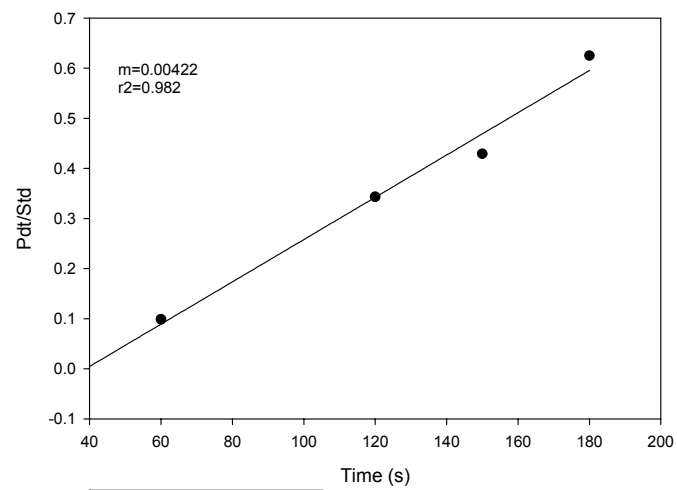

- Time $\mathrm{H} 1 \mathrm{vs} \mathrm{Pd} t / \mathrm{Std} \mathrm{H} 1$

DMSO H3

$\mathrm{m}=0.00391 \mathrm{r} 2=0.9906$

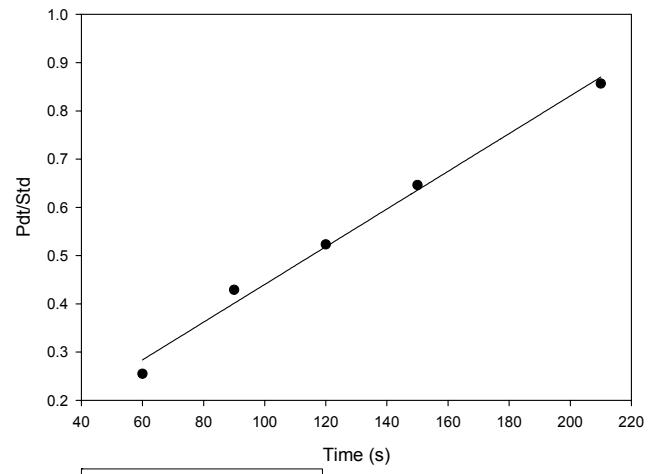

- Time $\mathrm{H} 3$ vs Pdt/Std H3
x column 2 vs y column 2

DMSO H5

$\mathrm{m}=0.00396 \mathrm{r} 2=0.9905$

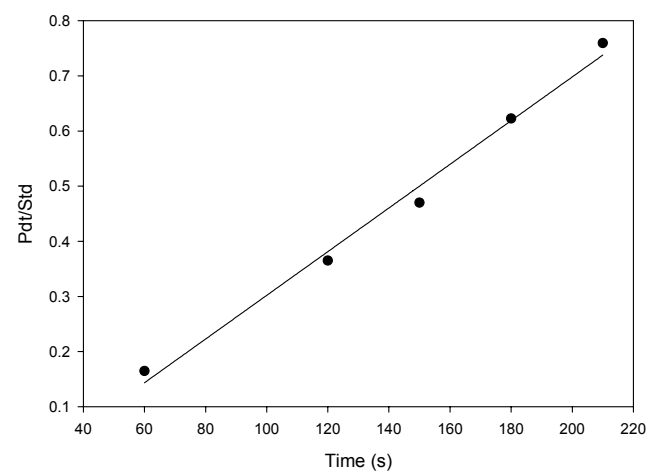

- Time H5 vs Pdt/Std H5
$\mathrm{x}$ column 4 vs y column

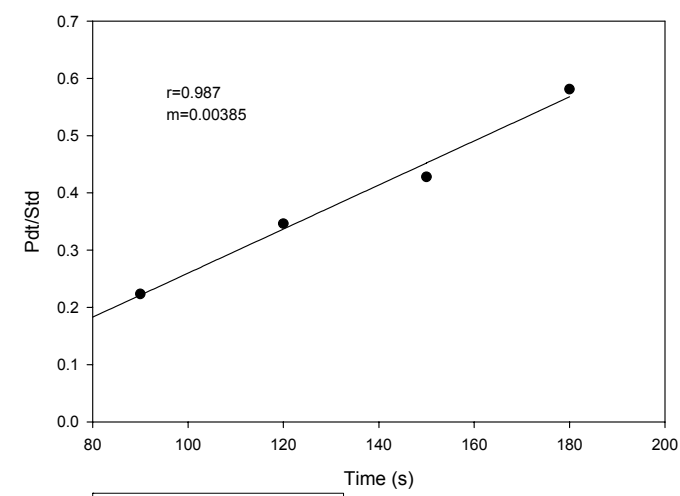

- Time H2 vs Pdt/Std H2 DMSO H4 $\mathrm{m}=0.00384 \mathrm{r} 2=0.9947$

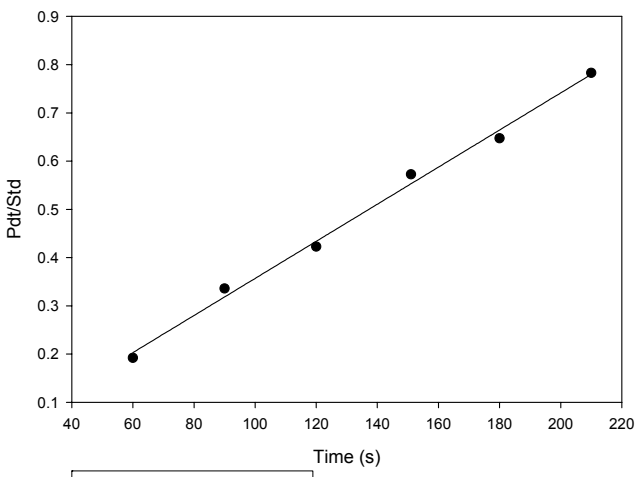

- Time $\mathrm{H} 4$ vs Pdt/Std H4
x column 3 vs y column 3 DMSO H6 $\mathrm{m}=0.00439 \mathrm{r} 2=0.9912$

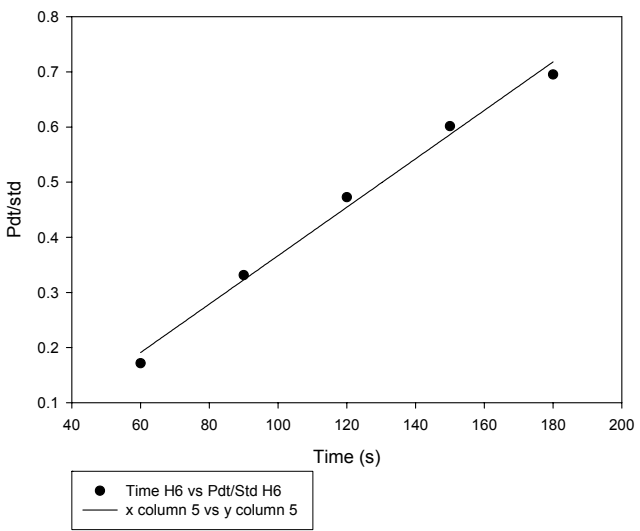


DMSO d1

$\mathrm{m}=0.000785 \mathrm{r} 2=0.9906$
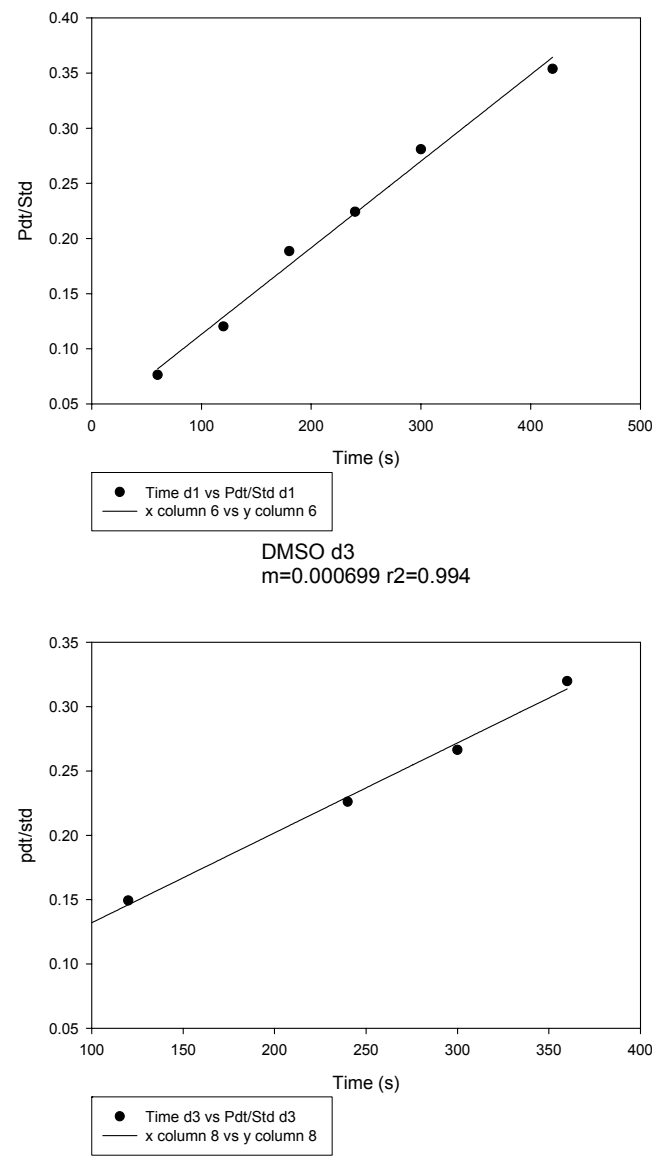

DMSO d2

$\mathrm{m}=0.000845 \mathrm{r} 2=0.9997$

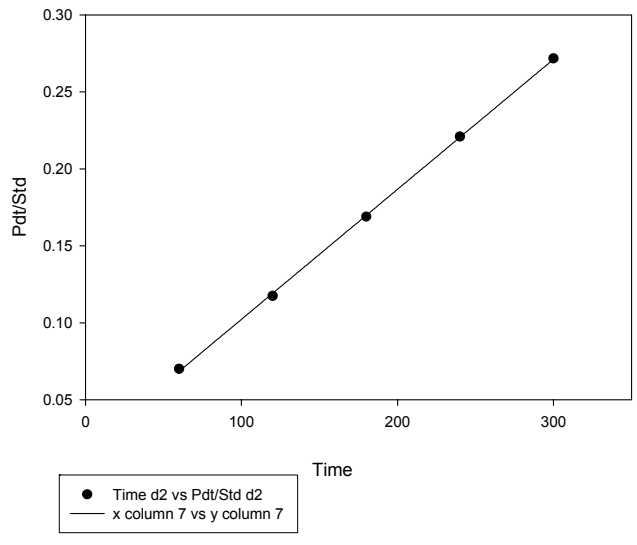


B. 4-Nitrobenzaldehyde in Acetonitrile

$\mathrm{K}_{\mathrm{H}}=8.28 \times 10^{-4} \pm 8.6 \times 10^{-6}$ (1\% error)

$\mathrm{K}_{\mathrm{D}}=1.96 \times 10^{-4} \pm 1.8 \times 10^{-6}(<1 \%$ error $)$

$\mathrm{KIE}=4.22 \pm 0.06$

$\mathrm{CH} 3 \mathrm{CN} \mathrm{H} 1$ (run 1) $\mathrm{m}=0.0008184 \mathrm{r} 2=1$
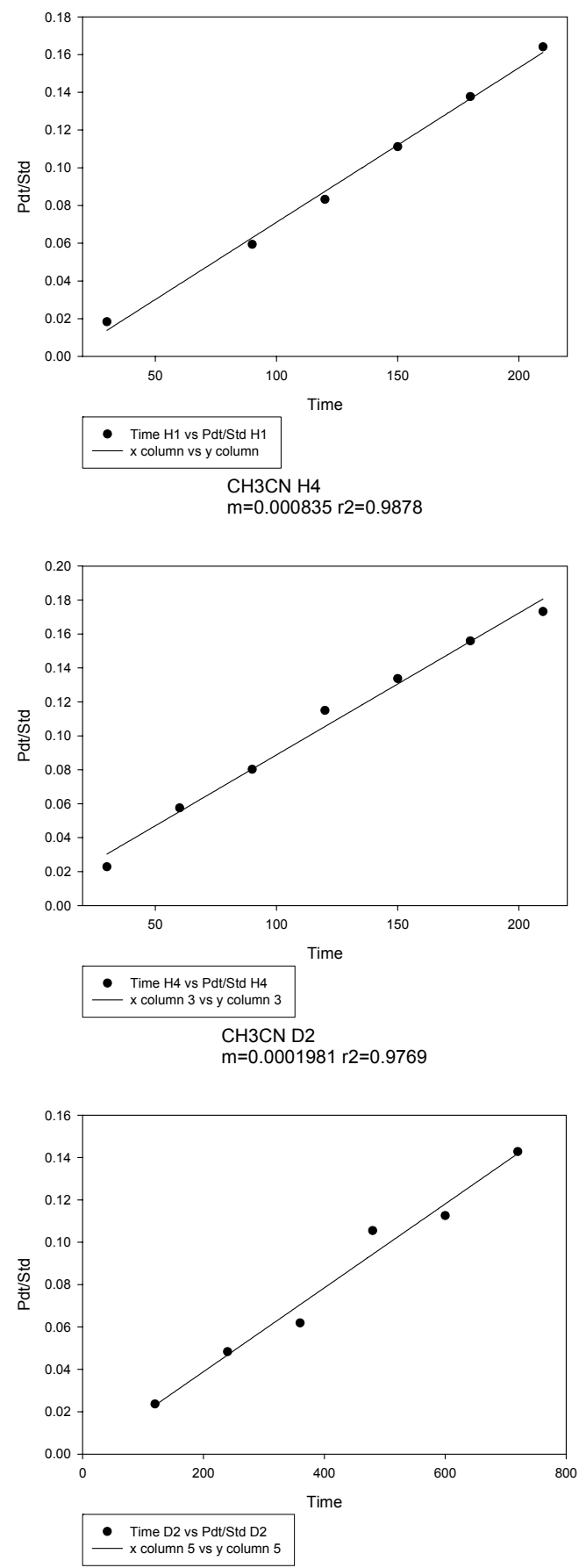

$\mathrm{CH} 3 \mathrm{CN} \mathrm{H} 3$ run 1 $\mathrm{m}=0.0008331 \mathrm{r} 2=0.994$
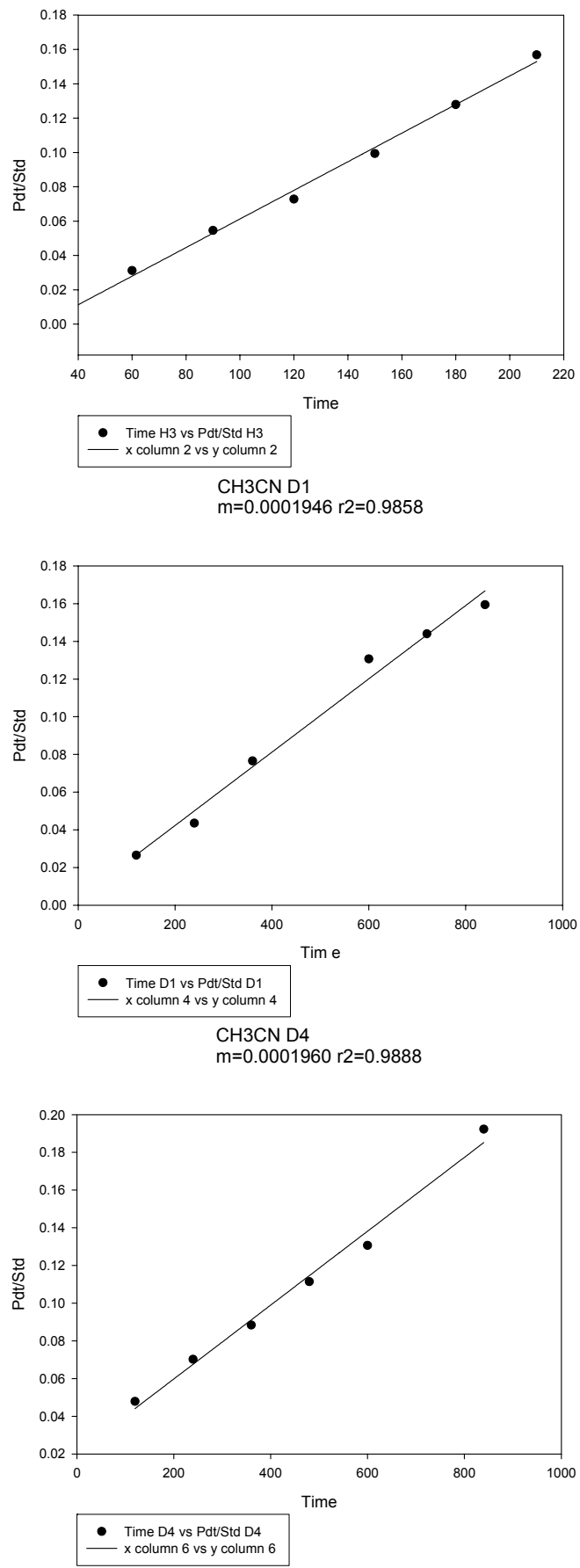
C. Nitrobenzaldehyde in DMF

$\mathrm{K}_{\mathrm{H}}=0.001125 \pm 4.384 \times 10^{-5}$ (3.90\% error)

$\mathrm{K}_{\mathrm{D}}=0.000391 \pm 1.530 \times 10^{-5}$ (3.80\% error)

$\mathrm{KIE}=2.88 \pm 0.16$

DMF H1

$\mathrm{m}=0.001083, \mathrm{r} 2=0.9995$
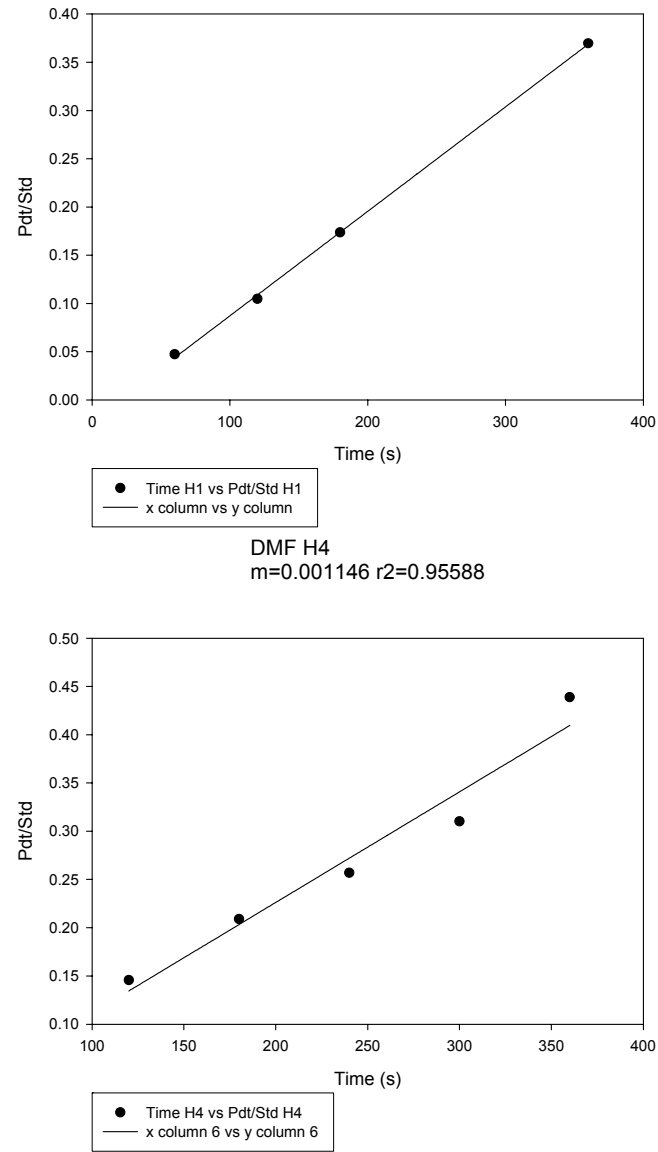

DMF H3

$\mathrm{m}=0.001176 \mathrm{r} 2=0.9719$
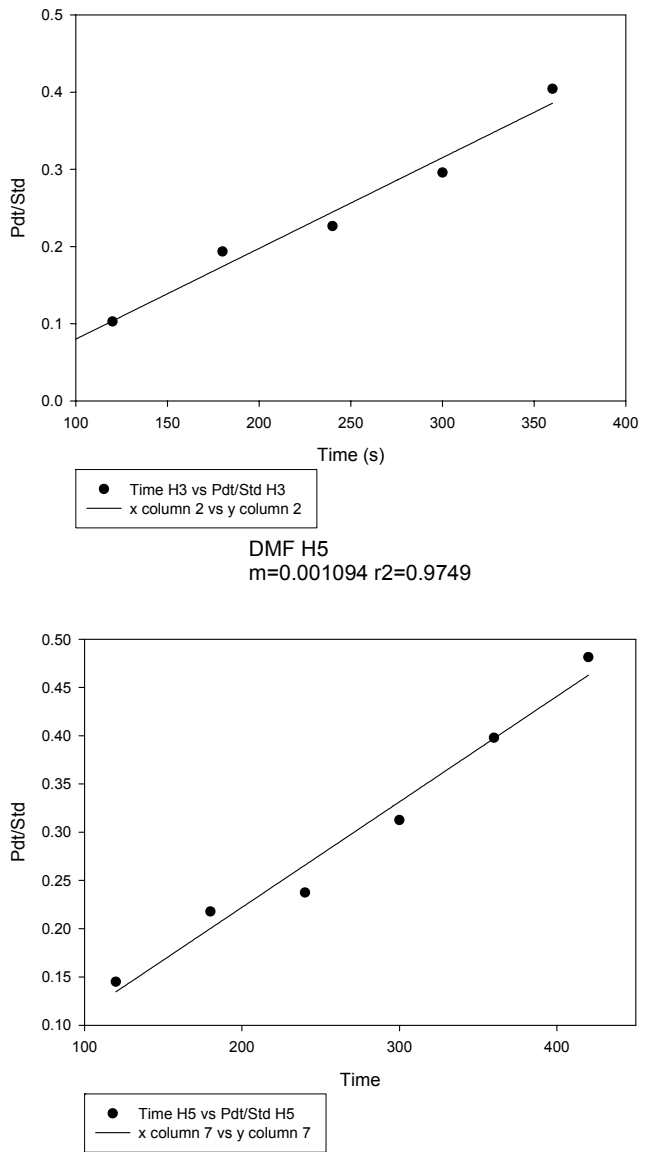
DMF D1

$\mathrm{m}=0.0004086 \mathrm{r} 2=0.985$
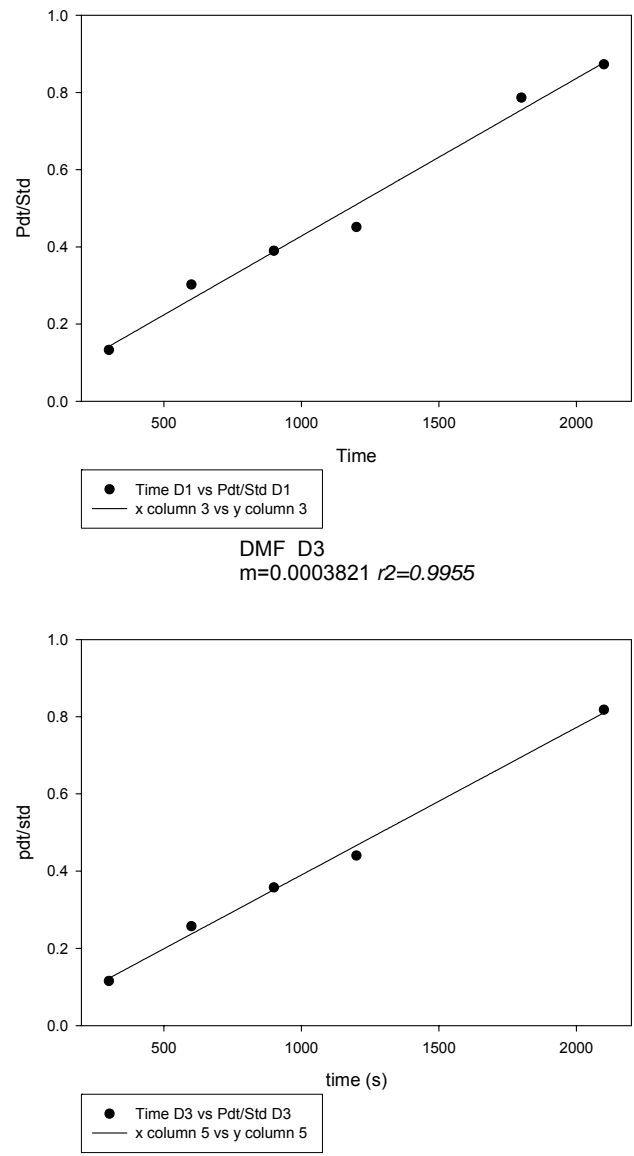

DMF D2- run 2

$\mathrm{m}=0.003817 \mathrm{r} 2=0.003817$

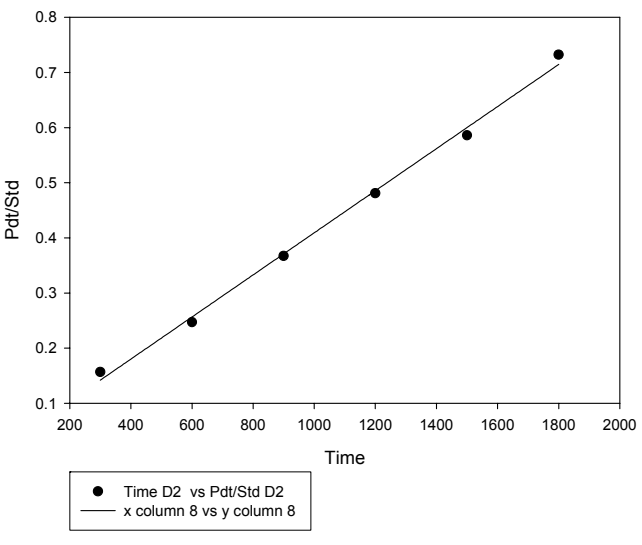




\section{4-Nitrobenzaldehyde in Chloroform}

$\mathrm{K}_{\mathrm{h}}=1.14 \times 10^{\wedge}-4 \pm 8.34 \times 10^{\wedge}-6$

$\mathrm{K}_{\mathrm{d}}=5.19 \times 10^{\wedge}-5 \pm 8.6 \times 10^{\wedge}-7$

$\mathrm{KIE}=2.19 \pm 0.16$

Chloroform $\mathrm{H} 2$

$m=0.0001234$ r2 $=0.9933$

Chloroform $\mathrm{H} 3$

$\mathrm{m}=0.0001125 \mathrm{r} 2=0.9933$
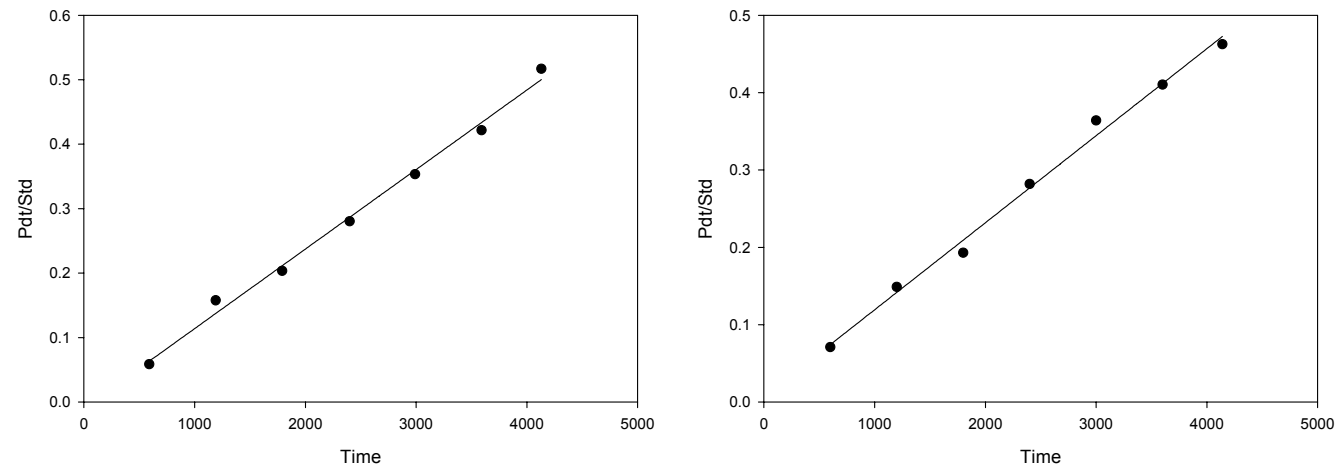

- Time H2 vs Pdt/Std H2
$\mathrm{x}$ column 3 vs y column 3

$\mathrm{CHCl} 3 \mathrm{H} 4$ Run 2

$\mathrm{m}=1.070 \times 10^{\wedge}-5 \mathrm{r} 2=0.985$

- Time H3 vs Pdt/Std H3
$-\mathrm{x}$ column 4 vs y column 4

$\mathrm{CHCl} 3 \mathrm{D} 1$

$\mathrm{m}=5.154 \times 10^{\wedge}-5 \mathrm{r} 2=0.9513$
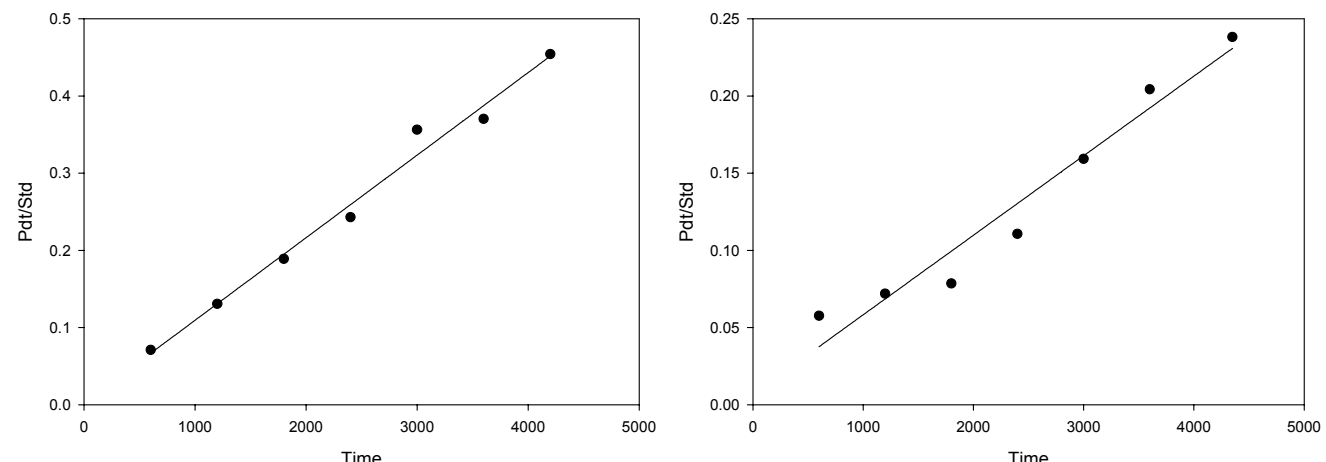

- Time H4 vs Pdt/Std H4
$-\mathrm{x}$ column 12 vs y column

- Time D1 vs Pdt/Std D1 $x$ column 5 vs y column 5

$\mathrm{CHCl} 3 \mathrm{D} 3$

$\mathrm{m}=5.119 \times 10^{\wedge}-5 \mathrm{r} 2=0.9701$

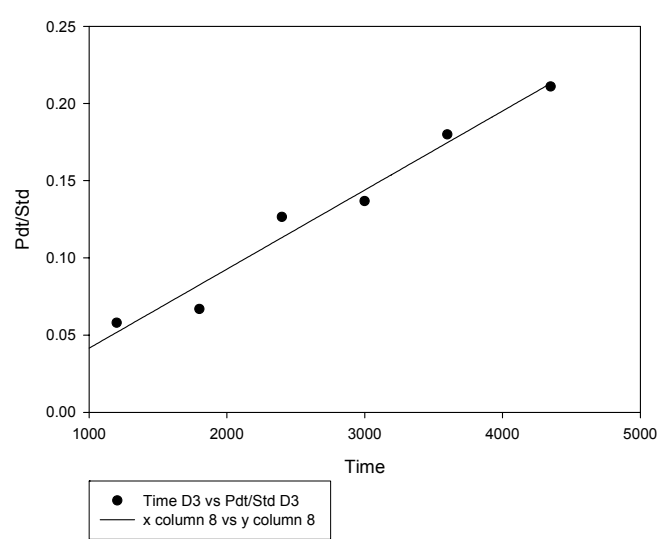

$\mathrm{CHCl} 3 \mathrm{D} 4 \mathrm{Run} 2$

$\mathrm{m}=5.283 \times 10^{\wedge}-5 \mathrm{r} 2=0.9934$

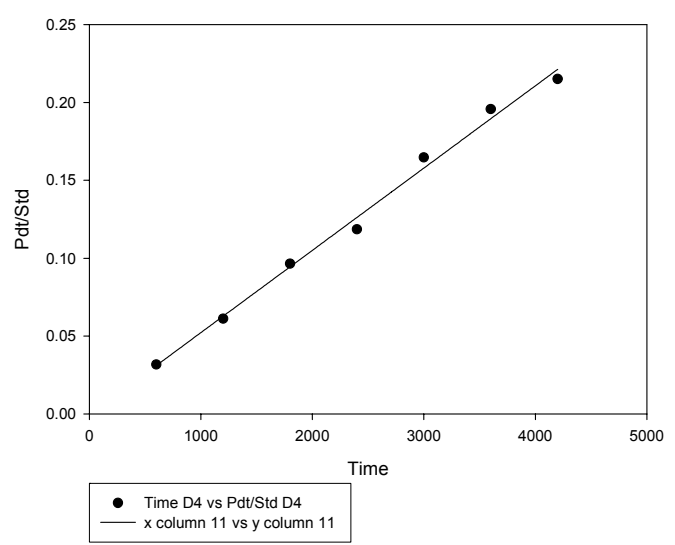


E. 4-Nitrobenzaldehyde in THF

$\mathrm{K}_{\mathrm{h}}=0.000174 \pm 0.00001$ (5.7\% error)

$\mathrm{K}_{\mathrm{d}}=0.0000736 \pm 1 \times 10^{-6}(1.4 \%$ error $)$

$\mathrm{KIE}=2.36 \pm 0.14$
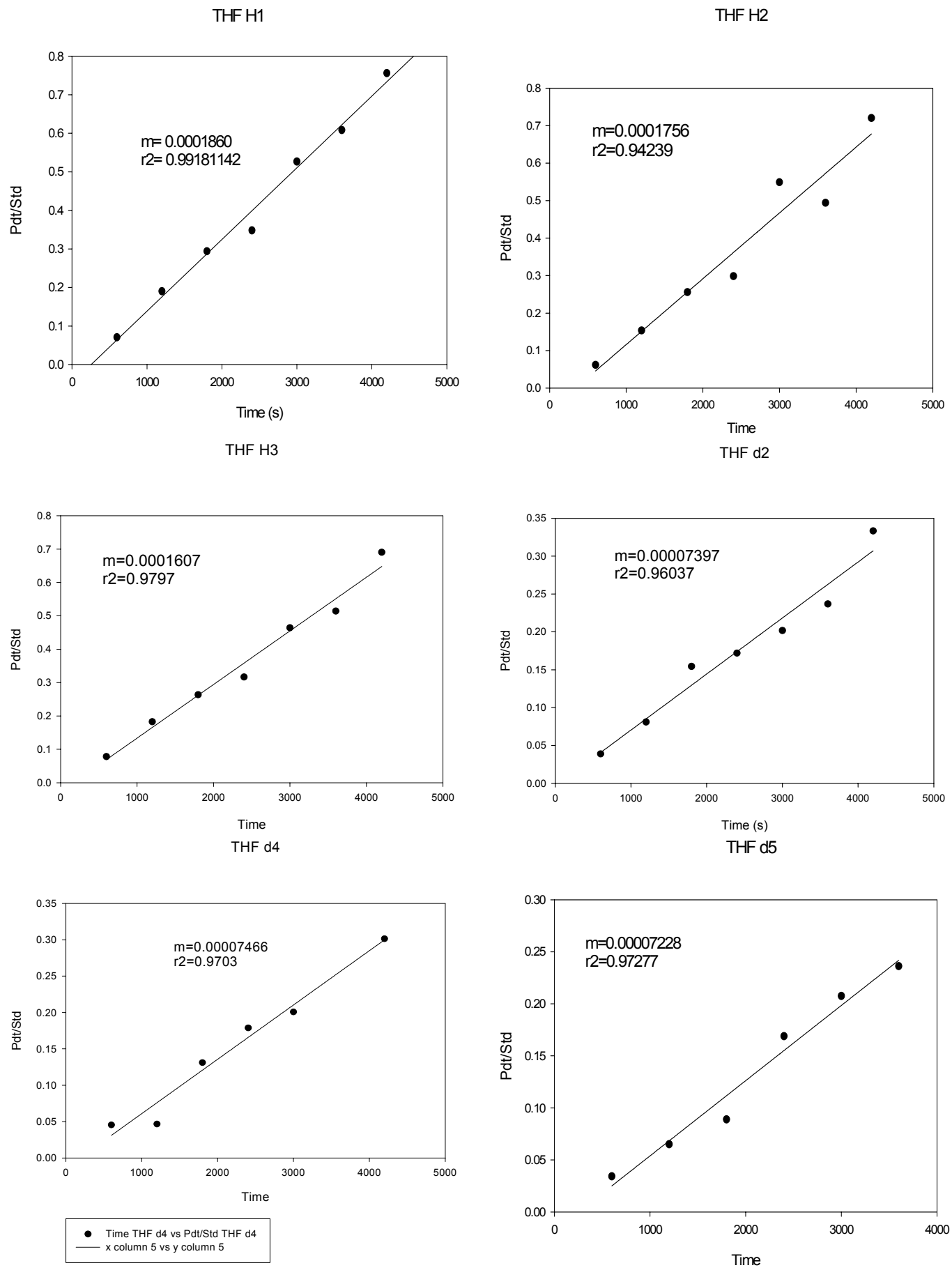


\section{Kinetic Isotope Effect at the Aldehyde Proton}
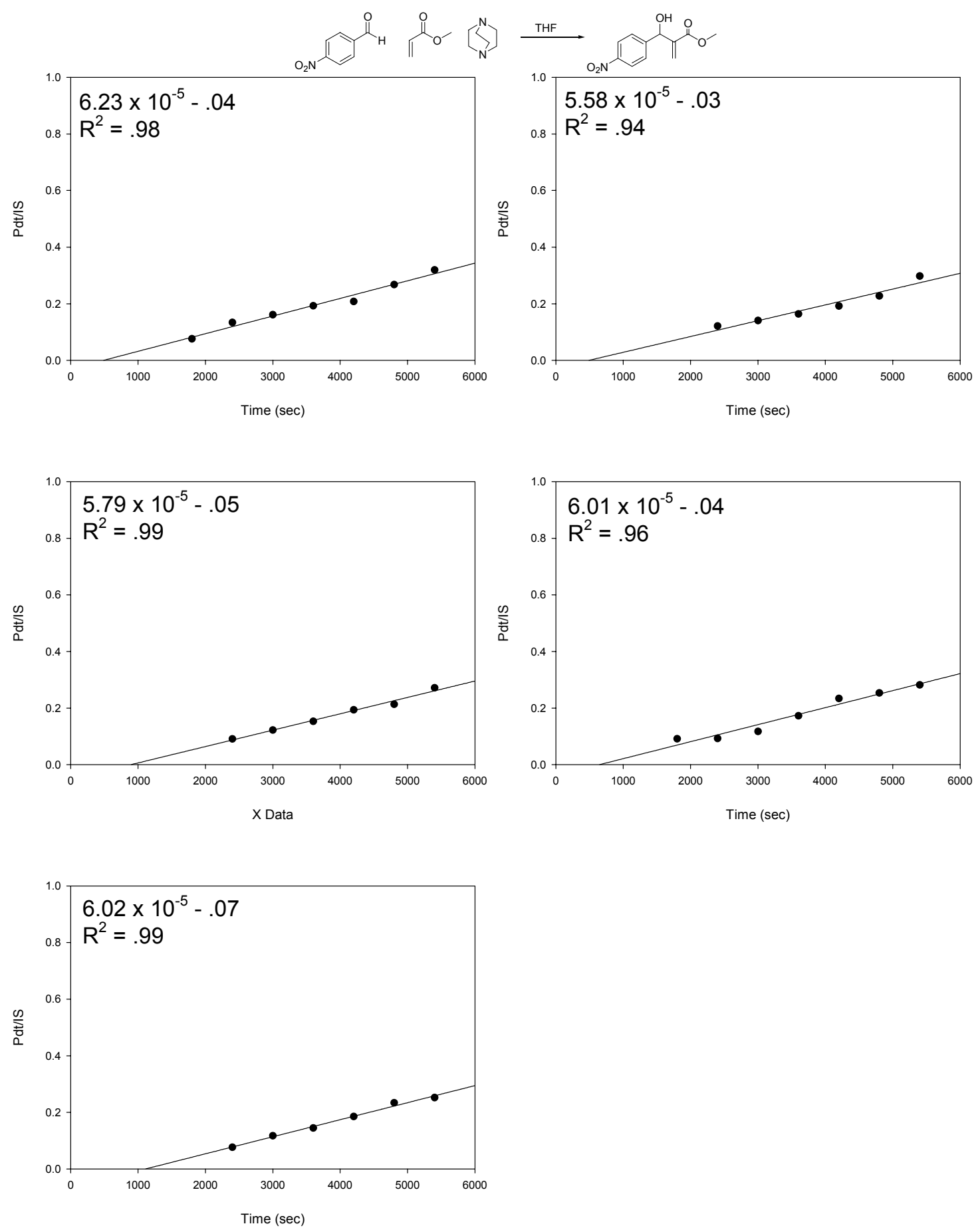

$$
\text { Avg. }=5.93 \times 10^{-5}+/-0.25 \times 10^{-5}(4 \%)
$$



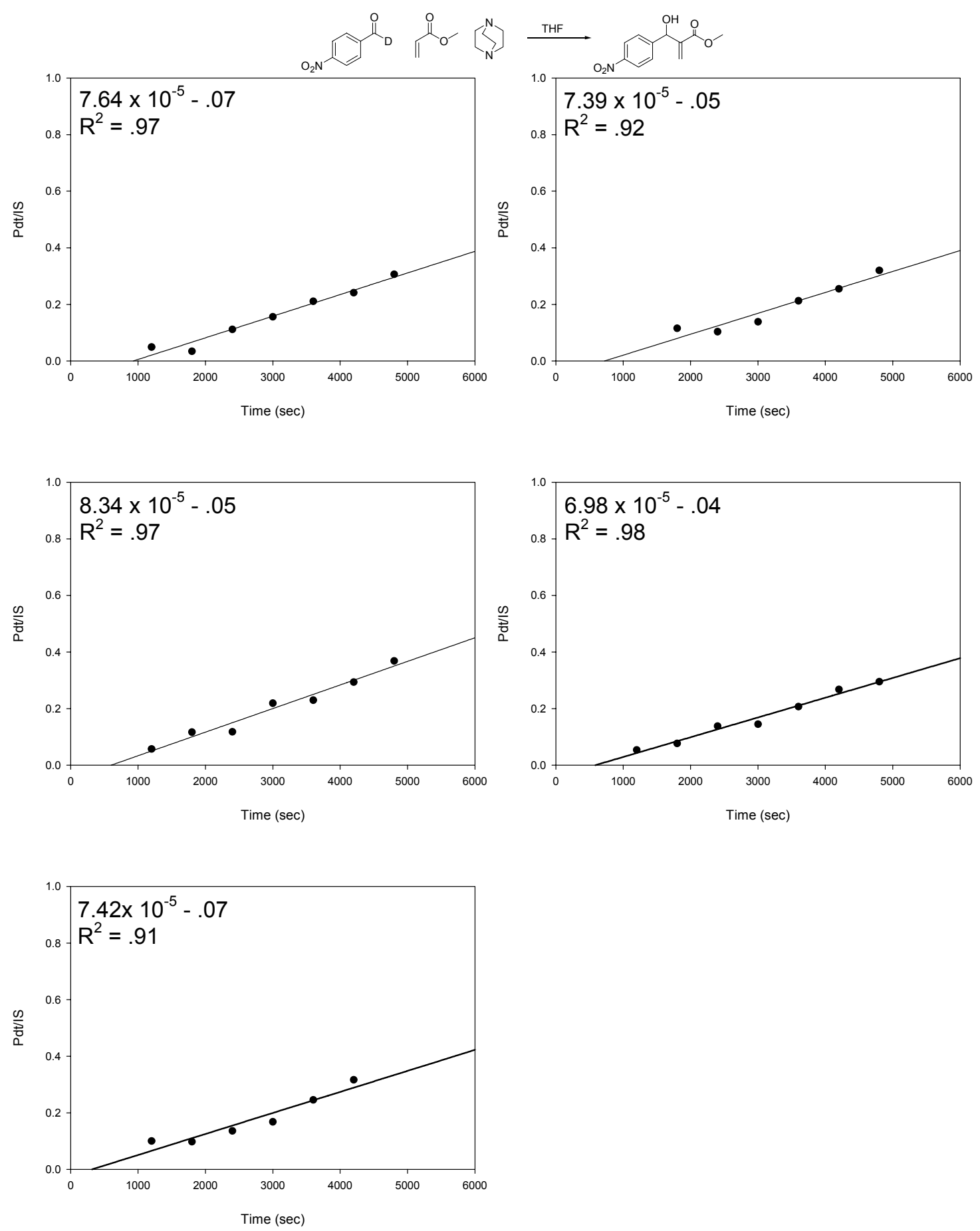

Avg. $=7.55 \times 10^{-5}+/-0.50 \times 10^{-5}(6.6 \%)$ 

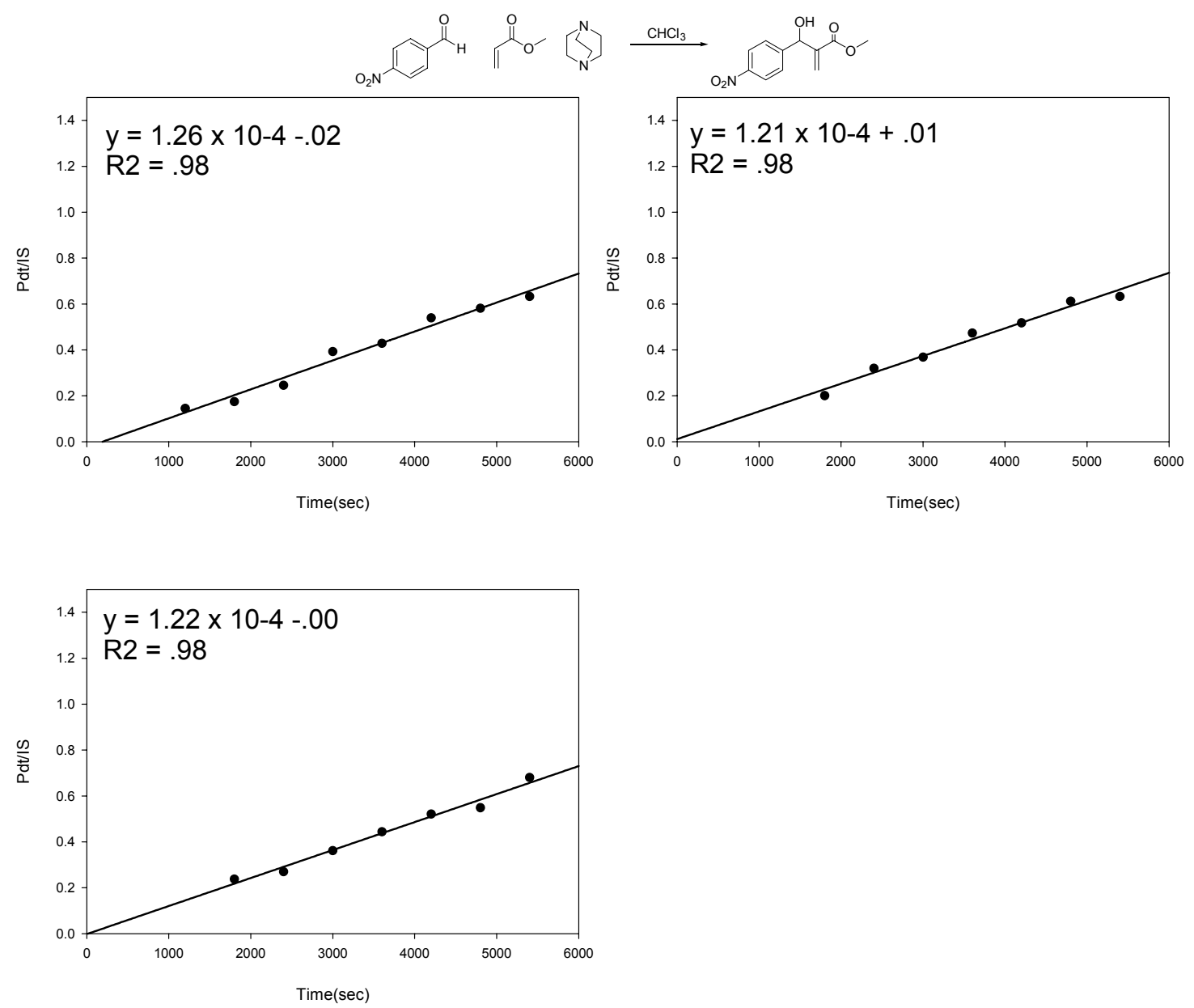

Avg. $=1.23 \times 10^{-4}+/-.03 \times 10^{-4}(2 \%)$ 


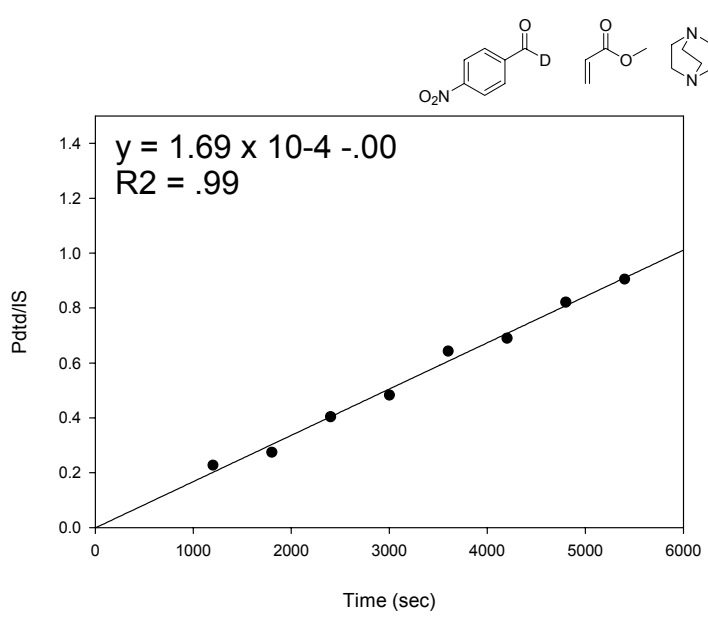

$\stackrel{\mathrm{CHCl}_{3}}{\longrightarrow}$
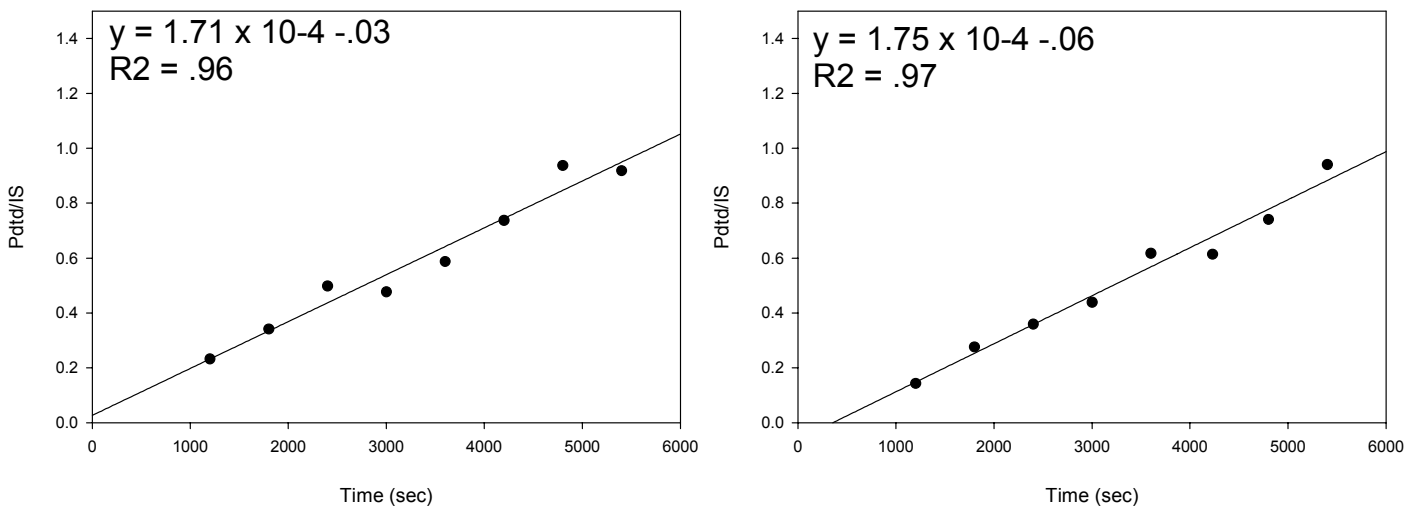

Avg. $=1.71 \times 10^{-4}+/-.03 \times 10^{-4}(1.7 \%)$ 

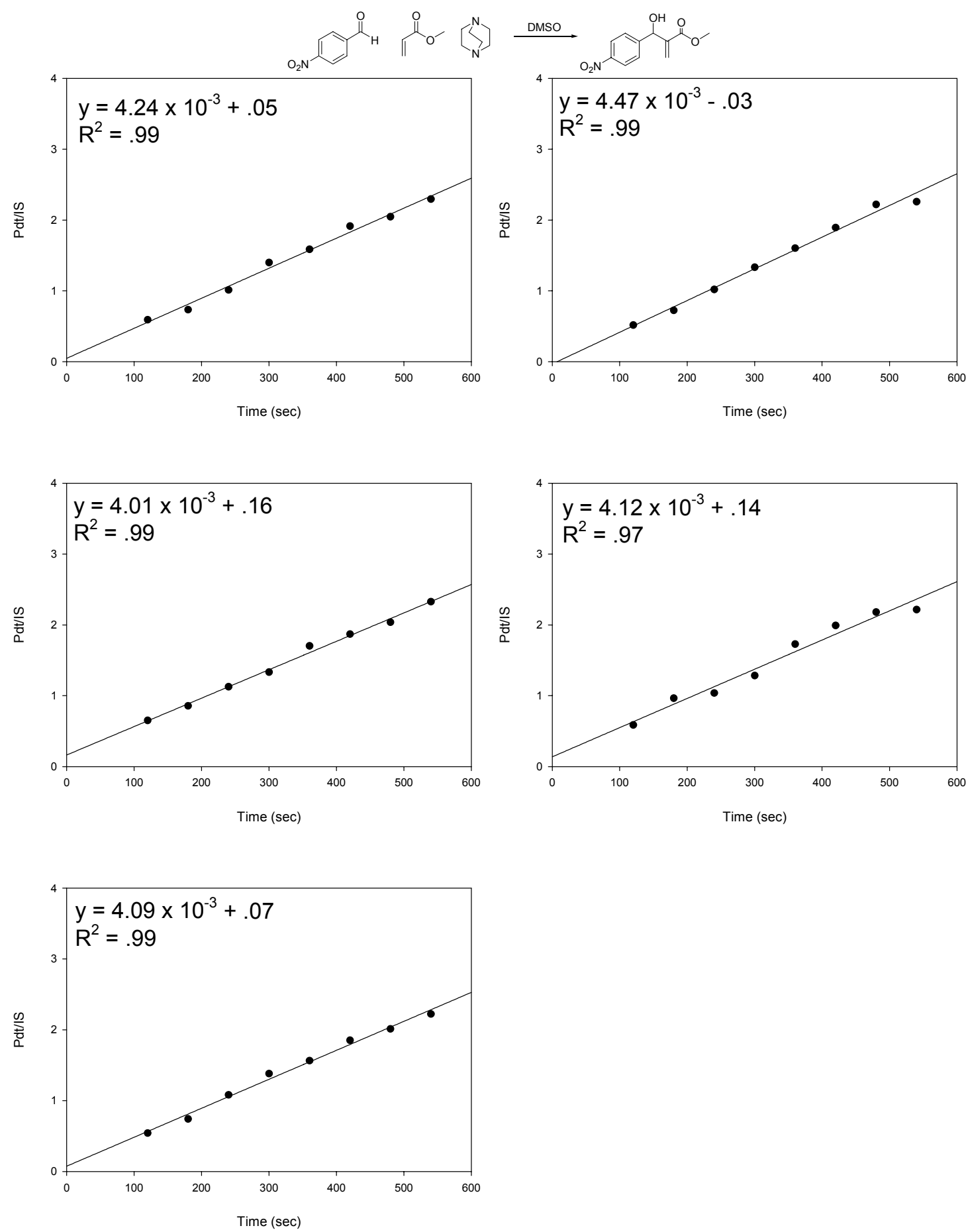

Avg. $=4.19 \times 10^{-3}+/-0.18 \times 10^{-3}(4 \%)$ 

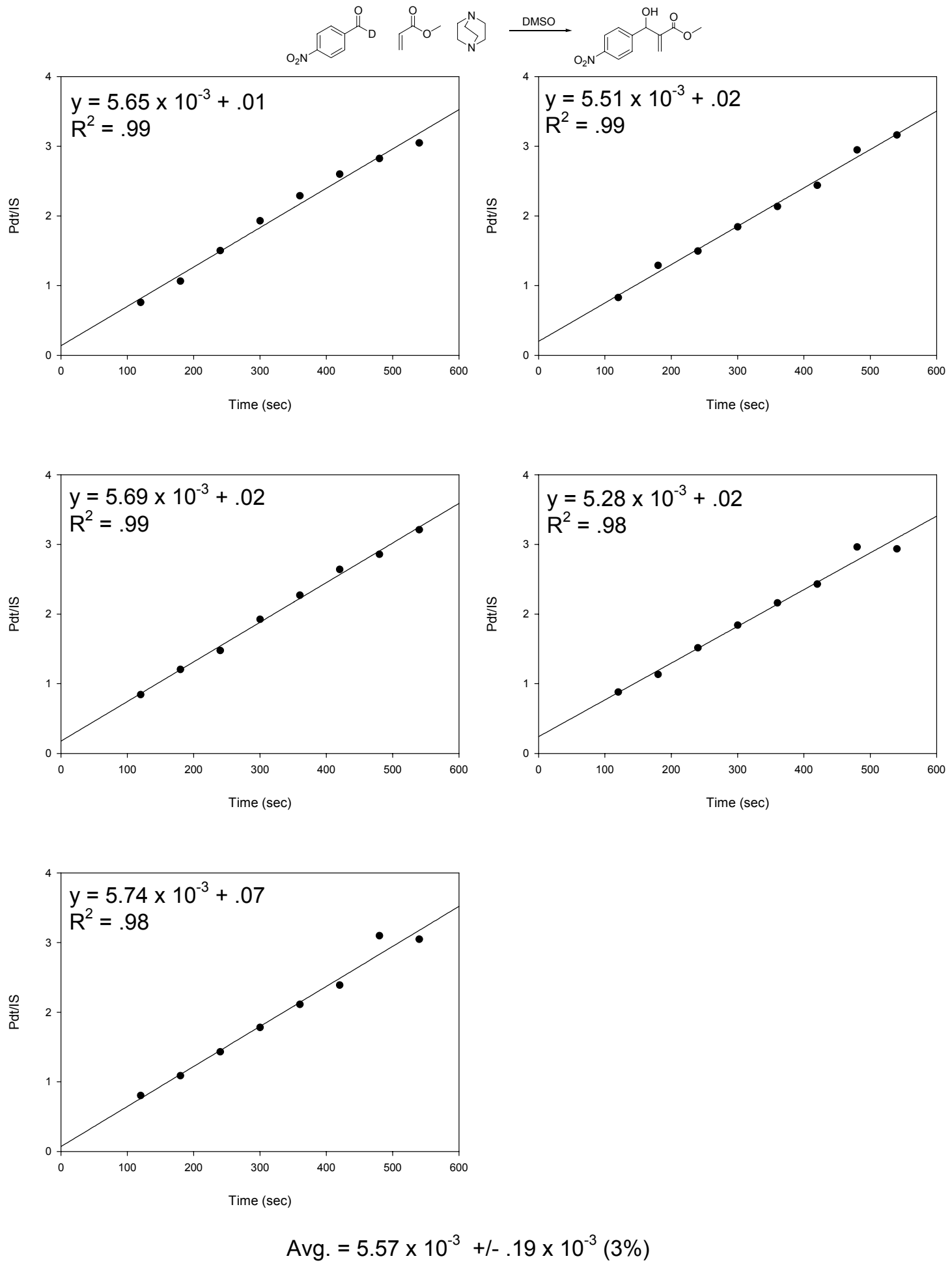

43 
IV. ${ }^{1}$ HNMR Spectrum of the Reaction Mixture at High Conversion

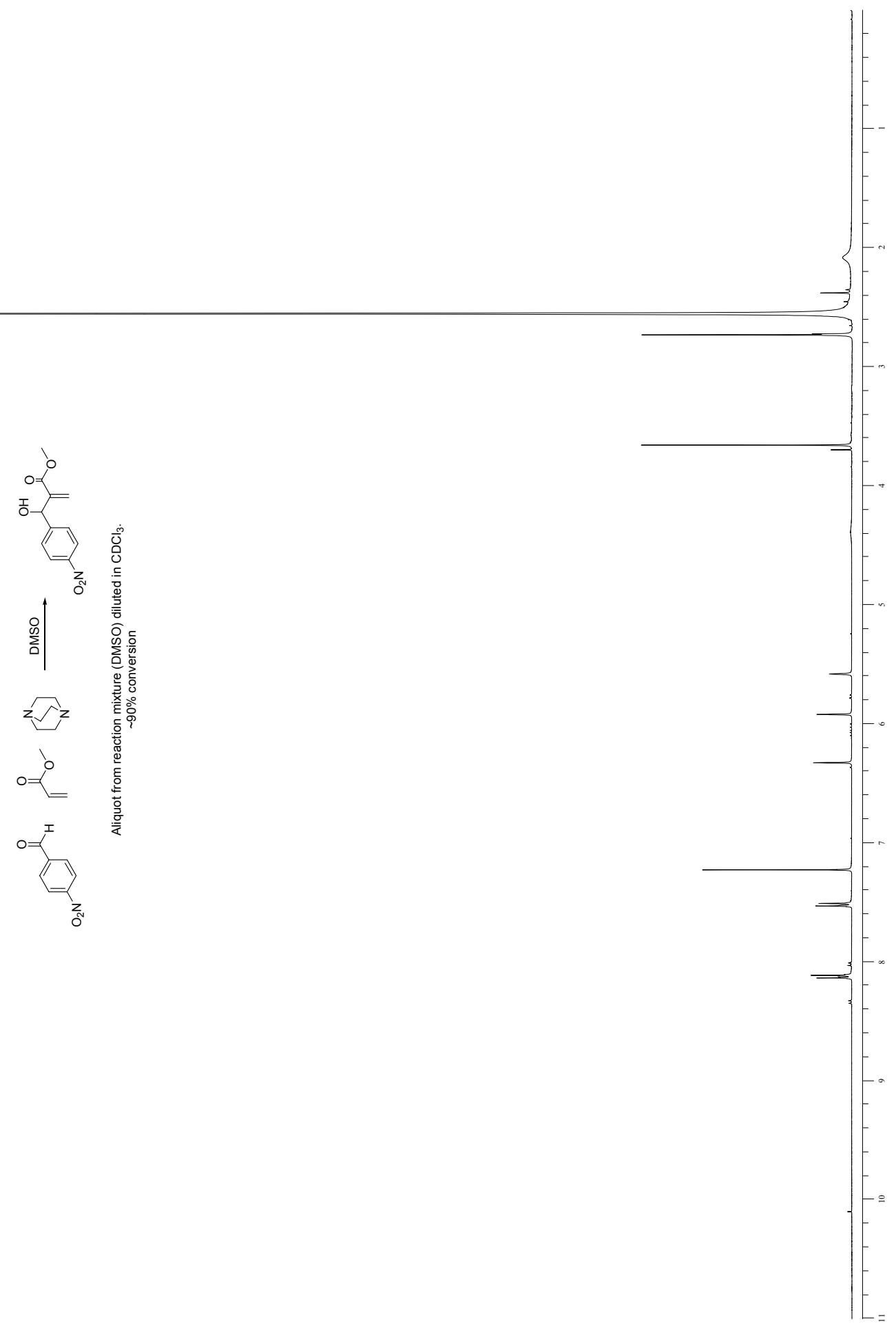

